\title{
Stepwise Square Integrable Representations: the Concept and Some Consequences
}

Joseph A. Wolf

\begin{abstract}
There are some new developments on Plancherel formula and growth of matrix coefficients for unitary representations of nilpotent Lie groups. These have several consequences for the geometry of weakly symmetric spaces and analysis on parabolic subgroups of real semisimple Lie groups, and to (infinite dimensional) locally nilpotent Lie groups. Many of these consequences are still under development. In this note I'll survey a few of these new aspects of representation theory for nilpotent Lie groups and parabolic subgroups.
\end{abstract}

\section{Introduction.}

There is a well developed theory of square integrable representations of nilpotent Lie groups [17]. It is based on the general representation theory of Kirillov [12] for connected nilpotent real Lie groups. A connected simply connected Lie group $N$ with center $Z$ is called square integrable if it has unitary representations $\pi$ whose coefficients $f_{u, v}(x)=\langle u, \pi(x) v\rangle$ satisfy $\left|f_{u, v}\right| \in \mathscr{L}^{2}(N / Z)$. If $N$ has one such square integrable representation then there is a certain polynomial function $P(\gamma)$ on the linear dual space $\mathfrak{z}^{*}$ of the Lie algebra of $Z$ that is key to harmonic analysis on $N$. Here $P(\gamma)$ is the Pfaffian of the antisymmetric bilinear form on $\mathfrak{n} / \mathfrak{z}$ given by $b_{\lambda}(x, y)=\lambda([x, y])$ where $\gamma=\left.\lambda\right|_{\mathfrak{z}}$. The square integrable representations of $N$ are certain easily-constructed representations $\pi_{\gamma}$ where $\gamma \in \mathfrak{z}^{*}$ with $P(\gamma) \neq 0$, Plancherel almost irreducible unitary representations of $N$ are square integrable, and up to an explicit constant $|P(\gamma)|$ is the Plancherel density of the unitary dual $\widehat{N}$ at $\pi_{\lambda}$. This theory has some interesting analytic consequences [26].

More recently there was a serious extension of that theory [28]. Under certain conditions, the nilpotent Lie group $N$ has a decomposition into subgroups that have

Joseph A. Wolf

Department of Mathematics, University of California, Berkeley, CA 94720-3840, USA, e-mail: jawolf@math.berkeley.edu 
square integrable representations, and the Plancherel formula then is synthesized explicitly in terms of the Plancherel formulae of those subgroups. In particular the extended theory applies to nilradicals of minimal parabolic subgroups [28]. With a minor technical adjustment it has just been extended to nilradicals of arbitrary real parabolics [32]. The consequences include explicit Plancherel and Fourier inversion formulas. Applications include analysis on minimal parabolic subgroups [29] and, more generally, on maximal amenable subgroups of parabolics [32], They also include analysis on commutative spaces, i.e. on Gelfand pairs [31]. We sketch some of these developments. Due to constraints of time and space we pass over many aspects of operator theory and orbit geometry, for example those described in [2], [3] and [4], related to stepwise square integrable representations.

In Section 2 we recall the basic facts [17], with a few extensions, on square integrable representations of nilpotent Lie groups. In Section 3 we recall the concept and main results for stepwise square integrable nilpotent Lie group.

In Section 4 we show how nilradicals of minimal parabolic subgroups have the required decomposition for stepwise square integrability. This is a construction based on concept of strongly orthogonal restricted roots.

In Section 5 we indicate the consequences for homogeneous compact nilmanifolds, and in Section 6 we mention the application to analysis on commutative nilmanifolds.

In Section 7 we start the extension of stepwise square integrability results from the nilradical $N$ of a minimal parabolic $P=M A N$ to various subgroups that contain $N$. This section concentrates on the subgroup $M N$ and takes advantage of principal orbit theory. That gives a sharp simplification to the Plancherel and Fourier Inversion formulae. In Section 8 we look at $P$ and its subgroup $A N$. They are not unimodular, so we introduce the Dixmier-Púkanszky operator $D$ whose semi-invariance balances that of the modular function. It is a key point for the Plancherel and Fourier Inversion formulae.

Sections 9 and 10 are a short discussion of work in progress on the extension of results from minimal parabolics to parabolics in general. There are two places where matters diverge from the minimal parabolic case. First, there is a technical adjustment to the definition of stepwise square integrable representation, caused by the fact that in the non-minimal case the restricted roots need not form a root system. Second, again for technical reasons, the explicit Plancherel Formula only comes through for the maximal amenable subgroups $U A N$ of $G$, and not for all of the parabolic.

This work was partially supported by a Simons Foundation grant and by the award of a Dickson Emeriti Professorship. It expands a talk at the 11-th International Workshop "Lie Theory and Its Applications in Physics" in Varna. My thanks to Prof. Vladimir Dobrev and the others on the organizing committee for hospitality at that Workshop. 


\section{Square Integrable Representations.}

Let $G$ be a unimodular locally compact group with center $Z$, and let $\pi$ be an irreducible unitary representation. We associate the central character $\chi_{\pi} \in \widehat{Z}$ by $\pi(z)=\chi_{\pi}(x) \cdot 1$ for $z \in Z$. Consider a matrix coefficient $f_{u, v}: x \mapsto\langle u, \pi(x) v\rangle$. Then $\left|f_{u, v}\right|$ is a well defined function on $G / Z$. Fix Haar measures $\mu_{G}$ on $G, \mu_{Z}$ on $Z$ and $\mu_{G / Z}$ on $G / Z$ such that $d \mu_{G}=d \mu_{Z} d \mu_{G / Z}$. The following results are well known.

Theorem 2.1. The following conditions on $\pi \in \widehat{G}$ are equivalent.

(1) There exist nonzero $u, v \in \mathscr{H}_{\pi}$ with $\left|f_{u, v}\right| \in \mathscr{L}^{2}(G / Z)$.

(2) $\left|f_{u, v}\right| \in \mathscr{L}^{2}(G / Z)$ for all $u, v \in \mathscr{H}_{\pi}$.

(3) $\pi$ is a discrete summand of the representation $\operatorname{Ind}_{Z}^{G}\left(\chi_{\pi}\right)$.

Theorem 2.2. If the conditions of Theorem 2.1 are satisfied for an irreducible $\pi \in$ $\widehat{G}$, then there is a number $\operatorname{deg} \pi>0$ such that

$$
\int_{G / Z} f_{u, v}(x) \overline{f_{u^{\prime}, v^{\prime}}(x)} d \mu_{G / Z}(x Z)=\frac{1}{\operatorname{deg} \pi}\left\langle u, u^{\prime}\right\rangle \overline{\left\langle v v^{\prime}\right\rangle}
$$

for all $u, u^{\prime}, v, v^{\prime} \in \mathscr{H}_{\pi}$. If $\pi_{1}, \pi_{2} \in \widehat{G}$ are inequivalent and satisfy the conditions of Theorem 2.1 and $\chi_{\pi_{1}}=\chi_{\pi_{2}}$, then

$$
\int_{G / Z}\left\langle u, \pi_{1}(x) v\right\rangle \overline{\left\langle u^{\prime}, \pi_{2}(x) v^{\prime}\right\rangle} d \mu_{G / Z}(x Z)=0
$$

for all $u, v \in \mathscr{H}_{\pi_{1}}$ and all $u^{\prime}, v^{\prime} \in \mathscr{H}_{\pi_{2}}$.

The main results of [17] shows exactly how this works for nilpotent Lie groups:

Theorem 2.3. Let $N$ be a connected simply connected Lie group with center $Z, \mathfrak{n}$ and $\mathfrak{z}$ their Lie algebras, and $\mathfrak{n}^{*}$ the linear dual space of $\mathfrak{n}$. Let $\lambda \in \mathfrak{n}^{*}$ and let $\pi_{\lambda}$ denote the irreducible unitary representation attached to $\operatorname{Ad}^{*}(N) \lambda$ by the Kirillov theory [12]. Then the following conditions are equivalent.

(1) $\pi_{\lambda}$ satisfies the conditions of Theorem 2.1

(2) The coadjoint orbit $\operatorname{Ad}^{*}(N) \lambda=\left\{v \in \mathfrak{n}^{*}|v|_{\mathfrak{z}}=\left.\lambda\right|_{\mathfrak{z}}\right.$.

(3) The bilinear form $b_{\lambda}(x, y)=\lambda([x, y])$ on $\mathfrak{n} / \mathfrak{z}$ is nondegenerate.

(4) The universal enveloping algebra $\mathscr{U}(\mathfrak{z})$ is the center of $\mathscr{U}(\mathfrak{n})$.

The Pfaffian polynomial $\operatorname{Pf}\left(b_{\lambda}\right)$ is a polynomial function $P\left(\left.\lambda\right|_{\mathfrak{z}}\right)$ on $\mathfrak{z}^{*}$, and the set of representations $\pi_{\lambda}$ for which these conditions hold, is parameterized by the set $\left\{\gamma \in \mathfrak{z}^{*} \mid P(\gamma) \neq 0\right\}$ (which is empty or Zariski open in $\mathfrak{z}^{*}$ ).

We will say that the connected simply connected Lie group $N$ is square integrable if there exists $\lambda \in \mathfrak{n}^{*}$ such that $\left.P\left(\left.\lambda\right|_{\mathfrak{z}}\right) \neq 0\right\}$. For convenience we will sometimes write $P(\lambda)$ for $P\left(\left.\lambda\right|_{\mathfrak{z}}\right)$ and $\pi_{\gamma}$ for $\pi_{\lambda}$ where $\gamma=\left.\lambda\right|_{\mathfrak{z}}$.

Theorem 2.4. Let $N$ be a square integrable connected simply connected Lie group with center $Z$. Then Plancherel measure on $\widehat{N}$ is concentrated on $\left\{\pi_{\lambda} \mid P(\lambda) \neq 0\right\}$, and there the Plancherel measure is given by the measure $|P(\lambda) d \lambda|$ on $\mathfrak{z}^{*}$ and the formal degree $\operatorname{deg} \pi_{\lambda}=\left|P\left(\left.\lambda\right|_{\mathfrak{z}}\right)\right|$. 
Given $\gamma \in \mathfrak{z}^{*}$ with $P(\gamma) \neq 0$ and a Schwartz class $(\mathscr{C}(N))$ function $f$ on $N$ we write $\mathscr{O}(\gamma)$ for the co-adjoint orbit $\operatorname{Ad}^{*}(N) \gamma=\gamma+\mathfrak{z}^{\perp}, f_{\gamma}$ for the restriction of $f \cdot \exp$ to $\mathscr{O}(\gamma)$, and $\widehat{f}_{\gamma}$ for the Fourier transform of $f_{\gamma}$ on $\mathscr{O}(\gamma)$.

Theorem 2.5. Let $N$ be a square integrable connected simply connected Lie group with center $Z$ and $f \in \mathscr{C}(N)$. If $\gamma \in \mathfrak{z}^{*}$ with $P(\gamma) \neq 0$ then the distribution character of $\pi_{\gamma}$ is given by

$$
\Theta_{\pi_{\gamma}}(f)=\operatorname{trace} \int_{N} f(x) \pi_{\gamma}(x) d \mu_{G}(x)=c^{-1}|P(\gamma)|^{-1} \int_{v \in \mathscr{O}(\gamma)} \widehat{f}_{\gamma} d \nu
$$

where $c=d ! 2^{d}$ and $d=\operatorname{dim}(\mathfrak{n} / \mathfrak{z}) / 2$ and $d v$ is ordinary Lebesgue measure on the affine space $\mathscr{O}(\gamma)$. The Fourier Inversion formula for $N$ is

$$
f(x)=c \int_{\mathfrak{z}^{*}} \Theta_{\gamma}\left(r_{x} f\right)|P(\gamma)| d \gamma \text { where }\left(r_{x} f\right)(y)=f(y x) \text { (right translate). }
$$

There also are multiplicity results on $\mathscr{L}^{2}(N / \Gamma)$ where $N$ is square integrable and $\Gamma$ is a discrete co-compact subgroup, but they are the same as in the stepwise square integrable case, so we postpone their description.

\section{Stepwise Square Integrability.}

In order to to go beyond square integrable nilpotent groups, we suppose that the connected simply connected nilpotent Lie group decomposes as

$$
N=L_{1} L_{2} \ldots L_{m-1} L_{m} \text { where }
$$

(a) each $L_{r}$ has unitary representations with coeff in $\mathscr{L}^{2}\left(L_{r} / Z_{r}\right)$,

(b) $N_{r}:=L_{1} L_{2} \ldots L_{r}$ is normal in $N$ with $N_{r}=N_{r-1} \rtimes L_{r}$,

(c) $\left[\mathfrak{l}_{r}, \mathfrak{z}_{s}\right]=0$ and $\left[\mathfrak{l}_{r}, \mathfrak{l}_{s}\right] \subset \mathfrak{v}$ for $r>s$ with $\mathfrak{l}_{r}=\mathfrak{z}_{r}+\mathfrak{v}_{r}$ where $\mathfrak{n}=\mathfrak{s}+\mathfrak{v}, \mathfrak{s}=\oplus \mathfrak{z} r$ and $\mathfrak{v}=\oplus \mathfrak{v}_{r}$.

We will use the following notation.

(a) $d_{r}=\frac{1}{2} \operatorname{dim}\left(\mathfrak{l}_{r} / \mathfrak{z}_{r}\right)$ so $\frac{1}{2} \operatorname{dim}(\mathfrak{n} / \mathfrak{s})=d_{1}+\cdots+d_{m}$,

$$
\text { and } c=2^{d_{1}+\cdots+d_{m}} d_{1} ! d_{2} ! \ldots d_{m} \text { ! }
$$

(b) $b_{\lambda_{r}}:(x, y) \mapsto \lambda([x, y])$ viewed as a bilinear form on $\mathfrak{l}_{r} / \mathfrak{z}_{r}$

(c) $S=Z_{1} Z_{2} \ldots Z_{m}=Z_{1} \times \cdots \times Z_{m}$ where $Z_{r}$ is the center of $L_{r}$

(d) $P$ : polynomial $P(\lambda)=\operatorname{Pf}\left(b_{\lambda_{1}}\right) \operatorname{Pf}\left(b_{\lambda_{2}}\right) \ldots \operatorname{Pf}\left(b_{\lambda_{m}}\right)$ on $\mathfrak{s}^{*}$

(e) $\mathfrak{t}^{*}=\left\{\lambda \in \mathfrak{s}^{*} \mid P(\lambda) \neq 0\right\}$

(f) $\pi_{\lambda} \in \widehat{N}$ for $\lambda \in \mathfrak{s}^{*}$ with $P(\lambda) \neq 0$, irreducible unitary representation of $N=L_{1} L_{2} \ldots L_{m}$ constructed as follows. 
Start with the representation $\pi_{\lambda_{1}} \in \widehat{N_{1}}$ specified by $\lambda_{1} \in \mathfrak{z}_{1}^{*}$ with $\operatorname{Pf}\left(b_{\lambda_{1}}\right) \neq 0$. Choose an invariant polarization $\mathfrak{p}_{1}^{\prime} \subset \mathfrak{n}_{2}$ for the linear functional $\lambda_{1}^{\prime} \in \mathfrak{n}_{2}^{*}$ that agrees with $\lambda_{1}$ on $\mathfrak{n}_{1}$ and vanishes on $\mathfrak{l}_{2}$. Since $L_{r}$ centralizes $S_{r-1}$, ad $\left.\mathrm{ad}^{*}\left(\mathfrak{l}_{2}\right)\left(\lambda_{1}^{\prime}\right)\right|_{\mathfrak{z}_{1}+\mathfrak{l}_{2}}=0$, so $\mathfrak{p}_{1}^{\prime}=\mathfrak{p}_{1}+\mathfrak{l}_{2}$ where $\mathfrak{p}_{1}$ is an invariant polarization for $\lambda_{1} \in \mathfrak{n}_{1}^{*}$. The associated representations are $\pi_{\lambda_{1}}^{\prime} \in \widehat{N_{2}}$ and $\pi_{\lambda_{1}} \in \widehat{N_{1}}$. Note that $N_{2} / P_{1}^{\prime}=N_{1} / P_{1}$, so the representation spaces $\mathscr{H}_{\pi_{\lambda_{1}}^{\prime}}=\mathscr{L}^{2}\left(N_{2} / P_{1}^{\prime}\right)=\mathscr{L}^{2}\left(N_{1} / P_{1}\right)=\mathscr{H}_{\pi_{\lambda_{1}}}$. In other words, $\pi_{\lambda_{1}}^{\prime}$ extends $\pi_{\lambda_{1}}$ to a unitary representation of $N_{2}$ on the same Hilbert space $\mathscr{H}_{\pi_{\lambda_{1}}}$, and $d \pi_{\lambda_{1}^{\prime}}\left(\mathfrak{z}_{2}\right)=0$. Now the Mackey Little Group method gives us

Lemma 3.1. The irreducible unitary representations of $N_{2}$, whose restrictions to $N_{1}$ are multiples of $\pi_{\lambda_{1}}$, are the $\pi_{\lambda_{1}}^{\prime} \widehat{\otimes} \gamma$ where $\gamma \in \widehat{L_{2}}=\widehat{N_{2} / N_{1}}$.

Given $\lambda_{2} \in \mathfrak{z}_{2}^{*}$ with $\operatorname{Pf}\left(b_{\lambda_{2}}\right) \neq 0$ we have $\pi_{\lambda_{2}} \in \widehat{L_{2}}$ with coefficients in $\mathscr{L}^{2}\left(L_{2} / Z_{2}\right)$. In the notation of Lemma 3.1 we define

$$
\pi_{\lambda_{1}+\lambda_{2}} \in \widehat{N_{2}} \text { by } \pi_{\lambda_{1}+\lambda_{2}}=\pi_{\lambda_{1}}^{\prime} \widehat{\otimes} \pi_{\lambda_{2}} \text {. }
$$

Proposition 3.2. The coefficients $f_{z, w}(x y)=\left\langle z, \pi_{\lambda_{1}+\lambda_{2}}(x y) w\right\rangle$ of $\pi_{\lambda_{1}+\lambda_{2}}$ belong to $\mathscr{L}^{2}\left(N_{2} / S_{2}\right)$, in fact satisfy $\left\|f_{z, w}\right\|_{\mathscr{L}^{2}\left(N_{r} / S_{r}\right)}^{2}=\frac{\|z\|^{2}\|w\|^{2}}{\operatorname{deg}\left(\pi_{\lambda_{1}}\right) \ldots \operatorname{deg}\left(\pi_{\lambda_{r}}\right)}$.

Proposition 3.2 starts a recursion using $N_{r+1}=N_{r} \rtimes L_{r+1}$. We fix nonzero $\lambda_{i} \in$ $\mathfrak{z}_{i}^{*}$ for $1 \leqq i \leqq r+1$, and we start with the representation $\pi_{\lambda_{1}+\cdots+\lambda_{r}}$ constructed step by step from the square integrable representations $\pi_{\lambda_{i}} \in \widehat{L_{i}}$ for $1 \leqq i \leqq r$. The representation space $\mathscr{H}_{\pi_{\lambda_{1}+\cdots+\lambda_{r}}}=\mathscr{H}_{\pi_{\lambda_{1}}} \widehat{\otimes} \ldots \widehat{\otimes} \mathscr{H}_{\pi_{\lambda_{r}}}$. The coefficients of $\pi_{\lambda_{1}+\cdots+\lambda_{r}}$ have absolute value in $\mathscr{L}^{2}\left(N_{r} / S_{r}\right)$. They satisfy

$$
\left\|f_{z, w}\right\|_{\mathscr{L}^{2}\left(N_{r} / S_{r}\right)}^{2}=\frac{\|z\|^{2}\|w\|^{2}}{\operatorname{deg}\left(\pi_{\lambda_{1}}\right) \ldots \operatorname{deg}\left(\pi_{\lambda_{r}}\right)} .
$$

Then $\pi_{\lambda_{1}+\cdots+\lambda_{r}}$ extends to a representation $\pi_{\lambda_{1}+\cdots+\lambda_{r}}^{\prime}$ of $L_{r+1}$ on the same Hilbert space $\mathscr{H}_{\pi_{\lambda_{1}+\cdots+\lambda_{r}}}$, and it satisfies $d \pi_{\lambda_{1}+\cdots+\lambda_{r}}^{\prime}\left(\mathfrak{z}_{r+1}\right)=0$. As in Lemma3.1,

Lemma 3.3. The irreducibles $\pi \in \widehat{N_{r+1}}$, whose restrictions to $N_{r}$ are multiples of $\pi_{\lambda_{1}+\cdots+\lambda_{r}}$, are the $\pi_{\lambda_{1}+\cdots+\lambda_{r}}^{\prime} \widehat{\otimes} \gamma$ where $\gamma \in \widehat{L_{r+1}}=\widehat{N_{r+1} / N_{r}}$.

As in Proposition 3.2 define $\pi_{\lambda_{1}+\cdots+\lambda_{r+1}}=\pi_{\lambda_{1}+\cdots+\lambda_{r}}^{\prime} \widehat{\otimes} \pi_{\lambda_{r+1}}$. Then

Proposition 3.4. The coefficients $f_{z, w}\left(x_{1} \ldots x_{r+1}\right)=\left\langle z, \pi_{\lambda_{1}+\cdots+\lambda_{r+1}}\left(x_{1} x_{2} \cdots x_{r+1}\right) w\right\rangle$ of $\pi_{\lambda_{1}+\cdots+\lambda_{r+1}}$ belong to $\mathscr{L}^{2}\left(N_{r+1} / S_{r+1}\right)$, in fact satisfy

$$
\left\|f_{z, w}\right\|_{\mathscr{L}^{2}\left(N_{r+1} / S_{r+1}\right)}^{2}=\frac{\|z\|\left\|^{2}\right\| w \|^{2}}{\operatorname{deg}\left(\pi_{\lambda_{1}}\right) \ldots \operatorname{deg}\left(\pi_{\lambda_{r+1}}\right)} .
$$

Since deg $\pi_{\lambda_{r}}=\left|\operatorname{Pf}\left(b_{\lambda_{r}}\right)\right|$, Proposition 3.4 is the recursion step for our construction. Passing to the end case $r+1=m$ we see that Plancherel measure is concentrated on $\left\{\pi_{\lambda} \mid \lambda \in \mathfrak{t}^{*}\right\}$. Using (5) (c) to see that conjugation by elements of $L_{s}$ has no effect on the $\operatorname{Pf}\left(b_{\lambda_{r}}\right)$ for $r<s$, we arrive at 
Theorem 3.5. Let $N$ be a connected simply connected nilpotent Lie group that satisfies (5). Then Plancherel measure for $N$ is concentrated on $\left\{\pi_{\lambda} \mid \lambda \in \mathfrak{t}^{*}, P(\lambda) \neq 0\right\}$. If $\lambda \in \mathfrak{t}^{*}, P(\lambda) \neq 0$ and $u, v \in \mathscr{H}_{\pi_{\lambda}}$, then the coefficient $f_{u, v}(x)=\left\langle u, \pi_{v}(x) v\right\rangle$ satisfies

$$
\left\|f_{u, v}\right\|_{\mathscr{L}^{2}(N / S)}^{2}=\|u\|^{2}\|v\|^{2} /|P(\lambda)|
$$

The distribution character $\Theta_{\pi_{\lambda}}: f \mapsto$ trace $\int_{G} f(x) \pi(x) d x$ of $\pi_{\lambda}$ is given by

$$
\Theta_{\pi_{\lambda}}(f)=c^{-1}|P(\lambda)|^{-1} \int_{\mathscr{O}(\lambda)} \widehat{f_{\lambda}}(\xi) d v_{\lambda}(\xi) \text { for } f \in \mathscr{C}(N)
$$

where $\mathscr{C}(N)$ is the Schwartz space, $\mathscr{O}(\lambda)=\operatorname{Ad}^{*}(N) \lambda=\mathfrak{s}^{\perp}+\lambda, f_{\lambda}$ is the lift $f_{\lambda}(\xi)=f(\exp (\xi)), \widehat{f_{\lambda}}$ is its classical Fourier transform, and $d v_{\lambda}$ is the translate of normalized Lebesgue measure from $\mathfrak{s}^{\perp}$ to $\operatorname{Ad}^{*}(N) \lambda$. Further,

$$
f(x)=c \int_{\mathfrak{t}^{*}} \Theta_{\pi_{\lambda}}\left(r_{x} f\right)|P(\lambda)| d \lambda \text { for } f \in \mathscr{C}(N) .
$$

Definition 3.6. The representations $\pi_{\lambda}$ of (6(f)) are the stepwise square integrable representations of $N$ relative to (5).

The left action $(l(x) f)(g)=f\left(x^{-1} g\right)$ and the right action $(r(y) f)(g)=f(g y)$ of $N$ on functions carries over to coefficients of $\pi$ as $l(x) r(y) f_{u, v}=f_{\pi(x) u, \pi(y) v}$. If $\pi=\pi_{\lambda}$ stepwise square integrable, $u, v \in \mathscr{H}_{\pi_{\lambda}}$ are $C^{\infty}$ vectors, and if $\Phi$ and $\Psi$ belong to the universal enveloping algebra $\mathscr{U}(\mathfrak{n})$, then $l(\Phi) r(\Psi) f_{u, v}=f_{d \pi(\Psi) u, d \pi(\Phi) v}$ is just another coefficient, $C^{\infty}$ and $\mathscr{L}^{2}(N / S)$. If $\zeta_{\lambda} \in \widehat{S}$ is the quasicentral character of $\pi_{\lambda}$ it follows that $f_{u, v}$ belongs to the relative Schwartz space $\mathscr{C}\left(N / S, \zeta_{\lambda}\right)$. In particular it follows that $\left|f_{u, v}\right| \in \mathscr{L}^{p}(N / S)$ for all $p \geqq 1$. Taking Schwartz class wave packets over $S$ of coefficient functions of stepwise square integrable representations of $N$ one can express the Plancherel formula of Theorem 3.5 in terms of coefficient functions.

\section{Nilradicals of Minimal Parabolics.}

Fix a real simple Lie group $G$, an Iwasawa decomposition $G=K A N$, and a minimal parabolic subgroup $Q=M A N$ in $G$. Let $m=\operatorname{rank}_{\mathbb{R}} G=\operatorname{dim}_{\mathbb{R}} A$. As usual, write $\mathfrak{k}$ for the Lie algebra of $K, \mathfrak{a}$ for the Lie algebra of $A$, and $\mathfrak{n}$ for the Lie algebra of $N$. Complete $\mathfrak{a}$ to a Cartan subalgebra $\mathfrak{h}$ of $\mathfrak{g}$. Then $\mathfrak{h}=\mathfrak{t}+\mathfrak{a}$ with $\mathfrak{t}=\mathfrak{h} \cap \mathfrak{k}$. Now we have root systems

$\Delta\left(\mathfrak{g}_{\mathbb{C}}, \mathfrak{h}_{\mathbb{C}}\right)$ : roots of $\mathfrak{g}_{\mathbb{C}}$ relative to $\mathfrak{h}_{\mathbb{C}}$ (ordinary roots),

$\Delta(\mathfrak{g}, \mathfrak{a})$ : roots of $\mathfrak{g}$ relative to $\mathfrak{a}$ (restricted roots),

$\Delta_{0}(\mathfrak{g}, \mathfrak{a})=\{\alpha \in \Delta(\mathfrak{g}, \mathfrak{a}) \mid 2 \alpha \notin \Delta(\mathfrak{g}, \mathfrak{a})\}$ (nonmultipliable). 
Here $\Delta(\mathfrak{g}, \mathfrak{a})$ and $\Delta_{0}(\mathfrak{g}, \mathfrak{a})$ are root systems in the usual sense. Any positive root system $\Delta^{+}\left(\mathfrak{g}_{\mathbb{C}}, \mathfrak{h}_{\mathbb{C}}\right) \subset \Delta\left(\mathfrak{g}_{\mathbb{C}}, \mathfrak{h}_{\mathbb{C}}\right)$ defines positive systems

$$
\begin{aligned}
& \Delta^{+}(\mathfrak{g}, \mathfrak{a})=\left\{\left.\gamma\right|_{\mathfrak{a}} \mid \gamma \in \Delta^{+}\left(\mathfrak{g}_{\mathbb{C}}, \mathfrak{h}_{\mathbb{C}}\right) \text { and }\left.\gamma\right|_{\mathfrak{a}} \neq 0\right\} \\
& \Delta_{0}^{+}(\mathfrak{g}, \mathfrak{a})=\Delta_{0}(\mathfrak{g}, \mathfrak{a}) \cap \Delta^{+}(\mathfrak{g}, \mathfrak{a}) .
\end{aligned}
$$

We can (and do) choose $\Delta^{+}(\mathfrak{g}, \mathfrak{h})$ so that

$\mathfrak{n}$ is the sum of the positive restricted root spaces and

if $\gamma \in \Delta\left(\mathfrak{g}_{\mathbb{C}}, \mathfrak{h}_{\mathbb{C}}\right)$ and $\left.\gamma\right|_{\mathfrak{a}} \in \Delta^{+}(\mathfrak{g}, \mathfrak{a})$ then $\gamma \in \Delta^{+}\left(\mathfrak{g}_{\mathbb{C}}, \mathfrak{h}_{\mathbb{C}}\right)$.

Two roots are called strongly orthogonal if their sum and their difference are not roots. Then they are orthogonal. The Kostant cascade construction is

$$
\begin{aligned}
& \beta_{1} \in \Delta^{+}(\mathfrak{g}, \mathfrak{a}) \text { is a maximal positive restricted root and } \\
& \beta_{r+1} \in \Delta^{+}(\mathfrak{g}, \mathfrak{a}) \text { is a maximum among the roots of } \Delta^{+}(\mathfrak{g}, \mathfrak{a}) \\
& \text { that are orthogonal to all } \beta_{i} \text { with } i \leqq r
\end{aligned}
$$

Then the $\beta_{r}$ are mutually strongly orthogonal. Each $\beta_{r} \in \Delta_{0}^{+}(\mathfrak{g}, \mathfrak{a})$, and $\beta_{1}$ is unique because $\Delta(\mathfrak{g}, \mathfrak{a})$ is irreducible. For $1 \leqq r \leqq m$ define

$$
\begin{aligned}
& \Delta_{1}^{+}=\left\{\alpha \in \Delta^{+}(\mathfrak{g}, \mathfrak{a}) \mid \beta_{1}-\alpha \in \Delta^{+}(\mathfrak{g}, \mathfrak{a})\right\} \text { and } \\
& \Delta_{r+1}^{+}=\left\{\alpha \in \Delta^{+}(\mathfrak{g}, \mathfrak{a}) \backslash\left(\Delta_{1}^{+} \cup \cdots \cup \Delta_{r}^{+}\right) \mid \beta_{r+1}-\alpha \in \Delta^{+}(\mathfrak{g}, \mathfrak{a})\right\} .
\end{aligned}
$$

Lemma 4.1. If $\alpha \in \Delta^{+}(\mathfrak{g}, \mathfrak{a})$, either $\alpha \in\left\{\beta_{1}, \ldots, \beta_{m}\right\}$ or $\alpha$ belongs to just one $\Delta_{r}^{+}$.

Lemma 4.2. $\Delta_{r}^{+} \cup\left\{\beta_{r}\right\}=\left\{\alpha \in \Delta^{+} \mid \alpha \perp \beta_{i}\right.$ for $i<r$ and $\left.\left\langle\alpha, \beta_{r}\right\rangle>0\right\}$. In particular, $\left[\mathfrak{l}_{r}, \mathfrak{l}_{s}\right] \subset \mathfrak{l}_{t}$ where $t=\min \{r, s\}$.

Lemma 4.1 shows that the Lie algebra $\mathfrak{n}$ of $N$ is the direct sum of its subspaces

$$
\mathfrak{l}_{r}=\mathfrak{g}_{\beta_{r}}+\sum_{\Delta_{r}^{+}} \mathfrak{g}_{\alpha} \text { for } 1 \leqq r \leqq m
$$

and Lemma 4.2 shows that $\mathfrak{n}$ has an increasing foliation by ideals

$$
\mathfrak{n}_{r}=\mathfrak{l}_{1}+\mathfrak{l}_{2}+\cdots+\mathfrak{l}_{r} \text { for } 1 \leqq r \leqq m .
$$

Now we will see that the corresponding group level decomposition $N=L_{1} L_{2} \ldots L_{m}$ and the semidirect product decompositions $N_{r}=N_{r-1} \rtimes L_{r}$ satisfy (5). Denote

$$
\begin{aligned}
& s_{\beta_{r}} \text { is the Weyl group reflection in } \beta_{r} \text { and } \\
& \sigma_{r}: \Delta(\mathfrak{g}, \mathfrak{a}) \rightarrow \Delta(\mathfrak{g}, \mathfrak{a}) \text { by } \sigma_{r}(\alpha)=-s_{\beta_{r}}(\alpha) .
\end{aligned}
$$

Note that $\sigma_{r}\left(\beta_{s}\right)=-\beta_{s}$ for $s \neq r,+\beta_{s}$ if $s=r$. If $\alpha \in \Delta_{r}^{+}$we still have $\sigma_{r}(\alpha) \perp \beta_{i}$ for $i<r$ and $\left\langle\sigma_{r}(\alpha), \beta_{r}\right\rangle>0$. If $\sigma_{r}(\alpha)<0$ then $\beta_{r}-\sigma_{r}(\alpha)>\beta_{r}$ contradicting maximality of $\beta_{r}$. Thus, using Lemma 4.2, $\sigma_{r}\left(\Delta_{r}^{+}\right)=\Delta_{r}^{+}$. 
Lemma 4.3. If $\alpha \in \Delta_{r}^{+}$then $\alpha+\sigma_{r}(\alpha)=\beta_{r}$. (It is possible that $\alpha=\sigma_{r}(\alpha)=\frac{1}{2} \beta_{r}$ when $\frac{1}{2} \beta_{r}$ is a root.). If $\alpha, \alpha^{\prime} \in \Delta_{r}^{+}$and $\alpha+\alpha^{\prime} \in \Delta(\mathfrak{g}, \mathfrak{a})$ then $\alpha+\alpha^{\prime}=\beta_{r}$.

Lemma 4.4. Let $\mathfrak{n}$ be a nilpotent Lie algebra, $\mathfrak{z}$ its center, and $\mathfrak{v}$ a vector space complement to $\mathfrak{z}$ in $\mathfrak{n}$. Suppose that $\mathfrak{v}=\mathfrak{u}+\mathfrak{u}^{\prime}, \mathfrak{u}=\sum \mathfrak{u}_{a}$ and $\mathfrak{u}^{\prime}=\sum \mathfrak{u}_{a}^{\prime}$, and $\mathfrak{z}=\sum \mathfrak{z} b$ with $\operatorname{dim}_{\mathfrak{z} b}=1$ in such a way that (i) each $\left[\mathfrak{u}_{a}, \mathfrak{u}_{a}\right]=0=\left[\mathfrak{u}_{a}^{\prime}, \mathfrak{u}_{a}^{\prime}\right]$, (ii) if $a_{1} \neq a_{2}$ then $\left[\mathfrak{u}_{a_{1}}, \mathfrak{u}_{a_{2}}^{\prime}\right]=0$ and (iii) for each a there is a nondegenerate pairing $\mathfrak{u}_{a} \otimes \mathfrak{u}_{a}^{\prime} \rightarrow \mathfrak{z}_{b_{a}}$, by $u \otimes u^{\prime} \mapsto\left[u, u^{\prime}\right]$. Then $\mathfrak{n}$ is a direct sum of Heisenberg algebras $\mathfrak{z}_{a}+\mathfrak{u}_{a}+\mathfrak{u}_{a}^{\prime}$ and the commutative algebra that is the sum of the remaining $\mathfrak{z} b$.

Now one runs through a number of special situations: (1) If $\mathfrak{g}$ is the split real form of $\mathfrak{g}_{\mathbb{C}}$ then each $L_{r}$ has square integrable representations. (2) If $\mathfrak{g}$ is simple but not absolutely simple then each $L_{r}$ has square integrable representations. (3) If $G$ is the quaternion special linear group $S L(n ; \mathbb{H})$ then $L_{1}$ has square integrable representations. (4) If $G$ is the group $E_{6, F_{4}}$ of collineations of the Cayley projective plane then $L_{1}$ has square integrable representations. (5) The group $L_{1}$ has square integrable representations. (6) If $\mathfrak{g}$ is absolutely simple then each $L_{r}$ has square integrable representations. Putting these together, Theorem 3.5 applies to nilradicals of minimal parabolic subgroups:

Theorem 4.5. Let $G$ be a real reductive Lie group, $G=K A N$ an Iwasawa decomposition, $\mathfrak{l}_{r}$ and $\mathfrak{n}_{r}$ the subalgebras of $\mathfrak{n}$ defined in (17) and 187, and $L_{r}$ and $N_{r}$ the corresponding analytic subgroups of $N$. Then the $L_{r}$ and $N_{r}$ satisfy (5). In particular, Plancherel measure for $N$ is concentrated on $\left\{\pi_{\lambda} \mid \lambda \in \mathfrak{t}^{*}\right\}$. If $\lambda \in \mathfrak{t}^{*}$, and if $u$ and $v$ belong to the representation space $\mathscr{H}_{\lambda}$ of $\pi_{\lambda}$, then the coefficient $f_{u, v}(x)=\left\langle u, \pi_{\lambda}(x) v\right\rangle$ satisfies $\left\|f_{u, v}\right\|_{\mathscr{L}^{2}(N / S)}^{2}=\frac{\|u\|^{2}\|v\|^{2}}{|P(\lambda)|}$. The distribution character $\Theta_{\pi_{\lambda}}$ of $\pi_{\lambda}$ satisfies $\Theta_{\pi_{\lambda}}(f)=c^{-1}|P(\lambda)|^{-1} \int_{\mathscr{O}(\lambda)} \widehat{f_{\lambda}}(\xi) d v_{\lambda}(\xi)$ for $f \in \mathscr{C}(N)$. Here $\mathscr{C}(N)$ is the Schwartz space, $\mathscr{O}(\lambda)$ is the coadjoint orbit $\operatorname{Ad}^{*}(N) \lambda=\mathfrak{s}^{\perp}+\lambda, f_{\gamma}$ is the lift $f_{\gamma}(\xi)=f(\exp (\xi))$ to $\mathfrak{s}^{\perp}+\lambda$, $\widehat{f}_{\gamma}$ is its classical Fourier transform, and $d v_{\lambda}$ is the translate of normalized Lebesgue measure from $\mathfrak{s}^{\perp}$ to $\operatorname{Ad}^{*}(N) \lambda$. The Plancherel formula on $N$ is $f(x)=c \int_{\mathfrak{t}^{*}} \Theta_{\pi_{\lambda}}\left(r_{x} f\right)|P(\lambda)| d \lambda$ for $f \in \mathscr{C}(N)$.

\section{Compact Nilmanifolds.}

Here are the basic facts on discrete uniform (i.e. co-compact) subgroups of connected simply connected nilpotent Lie groups. See [21, Chapter 2] for an exposition.

Proposition 5.1. The following are equivalent.

- $N$ has a discrete subgroup $\Gamma$ with $N / \Gamma$ compact.

- $N \cong N_{\mathbb{R}}$ where $N_{\mathbb{R}}$ is the group of real points in a unipotent linear algebraic group defined over the rational number field $\mathbb{Q}$

- $\mathfrak{n}$ has a basis $\left\{\xi_{j}\right\}$ for which the coefficients $c_{i, j}^{k}$ in $\left[\xi_{i}, \xi_{j}\right]=\sum c_{i, j}^{k} \xi_{k}$ are rational numbers. 
Under those conditions let $\mathfrak{n}_{\mathbb{Q}}$ denote the rational span of $\left\{\xi_{j}\right\}$ and let $\mathfrak{n}_{\mathbb{Z}}$ be the integral span. Then $\exp \left(\mathfrak{n}_{\mathbb{Z}}\right)$ generates a discrete subgroup $N_{\mathbb{Z}}$ of $N=N_{\mathbb{R}}$ and $N_{\mathbb{R}} / N_{\mathbb{Z}}$ is compact. Conversely, if $\Gamma$ is a discrete co-compact subgroup of $N$ then the $\mathbb{Z}-$ span of $\exp ^{-1}(\Gamma)$ is a lattice in $\mathfrak{n}$ for which any generating set $\left\{\xi_{j}\right\}$ is a basis of $\mathfrak{n}$ such that the coefficients $c_{i, j}^{k}$ in $\left[\xi_{i}, \xi_{j}\right]=\sum c_{i, j}^{k} \xi_{k}$ are rational numbers.

The conditions of Proposition 5.1 hold for the nilpotent groups studied in Section 4 there one can choose the basis $\left\{\xi_{j}\right\}$ of $\mathfrak{n}$ so that the $c_{i, j}^{k}$ are integers.

The basic facts on square integrable representations that occur in compact quotients $N / \Gamma$, as described in [17, Theorem 7], are

Proposition 5.2. Let $N$ be a connected simply connected nilpotent Lie group that has square integrable representations, and let $\Gamma$ a discrete co-compact subgroup. Let $Z$ be the center of $N$ and normalize the volume form on $\mathfrak{n} / \mathfrak{z}$ by normalizing Haar measure on $N$ so that $N / Z \Gamma$ has volume 1 . Let $P$ be the corresponding Pfaffian polynomial on $\mathfrak{z}^{*}$. Note that $\Gamma \cap Z$ is a lattice in $Z$ and $\exp ^{-1}(\Gamma \cap Z)$ is a lattice (denote it $\Lambda$ ) in $\mathfrak{z}$. That defines the dual lattice $\Lambda^{*}$ in $\mathfrak{z}^{*}$. Then a square integrable representation $\pi_{\lambda}$ occurs in $\mathscr{L}^{2}(N / \Gamma)$ if and only if $\lambda \in \Lambda^{*}$, and in that case $\pi_{\lambda}$ occurs with multiplicity $|P(\lambda)|$.

Definition 5.3. Let $N=N_{\mathbb{R}}$ be defined over $\mathbb{Q}$ as in Proposition 5.1 so we have a fixed rational form $N_{\mathbb{Q}}$. We say that a connected Lie subgroup $L \subset N$ is rational if $L \cap N_{\mathbb{Q}}$ is a rational form of $L$, in other words if $\mathfrak{l} \cap \mathfrak{n}_{\mathbb{Q}}$ contains a basis of $\mathfrak{l}$. We say that a decomposition (5) is rational if the subgroups $L_{r}$ and $N_{r}$ are rational.

The following is immediate from this definition.

Lemma 5.4. Let $N$ be defined over $\mathbb{Q}$ as in Proposition 5.1 with rational structure defined by a discrete co-compact subgroup $\Gamma$. If the decomposition (5) is rational then each $\Gamma \cap Z_{r}$ in $Z_{r}$, each $\Gamma \cap L_{r}$ in $L_{r}$, each $\Gamma \cap S_{r}$ in $S_{r}$, and each $\Gamma \cap N_{r}$ in $N_{r}$, is a discrete co-compact subgroup defining the same rational structure as the one defined by its intersection with $N_{\mathbb{Q}}$.

Now assume that $N$ and $\Gamma$ satisfy the rationality conditions of Lemma 5.4 Then for each $r, Z_{r} \cap \Gamma$ is a lattice in the center $Z_{r}$ of $L_{r}$, and $\Lambda_{r}:=\log \left(Z_{r} \cap \Gamma\right)$ is a lattice in its Lie algebra $\mathfrak{z}_{r}$. That defines the dual lattice $\Lambda_{r}^{*}$ in $\mathfrak{z}_{r}^{*}$. We normalize the Pfaffian polynomials on the $\mathfrak{z}_{r}^{*}$, and thus the polynomial $P$ on $\mathfrak{s}^{*}$, by requiring that the $N_{r} /\left(S_{r} \cdot\left(N_{r} \cap \Gamma\right)\right)$ have volume 1 .

Theorem 5.5. Let $\lambda \in \mathfrak{t}^{*}$. Then a stepwise square integrable representation $\pi_{\lambda}$ of $N$ occurs in $\mathscr{L}^{2}(N / \Gamma)$ if and only if each $\lambda_{r} \in \Lambda_{r}^{*}$, and in that case the multiplicity of $\pi_{\lambda}$ on $\mathscr{L}^{2}(N / \Gamma)$ is $|P(\lambda)|$.

\section{Commutative Spaces.}

A commutative space $X=G / K$, or equivalently a Gelfand pair $(G, K)$, consists of a locally compact group $G$ and a compact subgroup $K$ such that the convolution algebra $\mathscr{L}^{1}(K \backslash G / K)$ is commutative. When $G$ is a connected Lie group it is equivalent 
to say that the algebra $\mathscr{D}(G, K)$ of $G$-invariant differential operators on $G / K$ is commutative. We say that the commutative space $G / K$ is a commutative nilmanifold if it is a nilmanifold in the sense that some nilpotent analytic subgroup $N$ of $G$ acts transitively. When $G / K$ is connected and simply connected it follows that $N$ is the nilradical of $G$, that $N$ acts simply transitively on $G / K$, and that $G$ is the semidirect product group $N \rtimes K$, so that $G / K=(N \rtimes K) / K$. In this section we study the role of square integrability and stepwise square integrability for commutative nilmanifolds $G / K=(N \rtimes K) / K$.

The cases where $G / K$ and $(G, K)$ are irreducible in the sense that $[\mathfrak{n}, \mathfrak{n}]$ (which must be central) is the center of $\mathfrak{n}$ and $K$ acts irreducibly on $\mathfrak{n} /[\mathfrak{n}, \mathfrak{n}]$, have been classified by E. B. Vinberg ([22], [23]). See [26, $\{13.4 B]$ for the Lie algebra structure $\mathfrak{v} \times \mathfrak{v} \rightarrow \mathfrak{z}$. The classification of commutative nilmanifolds is based on Vinberg's work and was completed by O. Yakimova in [34] and [35].

It turns out that almost all commutative manifolds correspond to nilpotent groups that are square integrable. The exceptions are those with a certain direct factor, and in those cases the nilpotent group is stepwise square integrable in two steps, so in those cases the Plancherel formula follows directly from the general result above. See [31] for the details.

\section{Minimal Parabolics: Subgroup $M N$.}

Fix an Iwasawa decomposition $G=K A N$ for a simple Lie group $G$ and the minimal parabolic subgroup $Q=M A N$. As usual, write $\mathfrak{k}$ for the Lie algebra of $K$, $\mathfrak{a}$ for the Lie algebra of $A, \mathfrak{m}$ for the Lie algebra of $M$, and $\mathfrak{n}$ for the Lie algebra of $N$. Complete $\mathfrak{a}$ to a Cartan subalgebra $\mathfrak{h}$ of $\mathfrak{g}$. Then we have root systems $\Delta\left(\mathfrak{g}_{\mathbb{C}}, \mathfrak{h}_{\mathbb{C}}\right)$, $\Delta(\mathfrak{g}, \mathfrak{a})$ and $\Delta_{0}(\mathfrak{g}, \mathfrak{a})$ described in (12). $M$ is the centralizer of $A$ in $K$. Write ${ }^{0}$ for identity component; then $Q^{0}=M^{0} A N$.

Recall the Pf-nonsingular set $\mathrm{t}^{*}=\left\{\lambda \in \mathfrak{s}^{*} \mid \operatorname{Pf}\left(b_{\lambda}\right) \neq 0\right\}$ of $(6 \mathrm{e})$; so $\operatorname{Ad}^{*}(M) \mathfrak{t}^{*}=$ $\mathfrak{t}^{*}$. Further, if $\lambda \in \mathfrak{t}^{*}$ and $c \neq 0$ then $c \lambda \in \mathfrak{t}^{*}$, in fact $\operatorname{Pf}\left(b_{c \lambda}\right)=c^{\operatorname{dim}(\mathfrak{n} / \mathfrak{s}) / 2} \operatorname{Pf}\left(b_{\lambda}\right)$.

Fix an $M$-invariant inner product $(\mu, v)$ on $\mathfrak{s}^{*}$. $\operatorname{So~} \operatorname{Ad}^{*}(M)$ preserves each sphere $\mathfrak{s}_{t}^{*}=\left\{\lambda \in \mathfrak{s}^{*} \mid(\lambda, \lambda)=t^{2}\right\}$. Two orbits $\operatorname{Ad}^{*}(M) \mu$ and $\operatorname{Ad}^{*}(M) v$ are of the same orbit type if the isotropy subgroups $M_{\mu}$ and $M_{\nu}$ are conjugate, and an orbit is principal if all nearby orbits are of the same type. Since $M$ and $\mathfrak{s}_{t}^{*}$ are compact, there are only finitely many orbit types of $M$ on $\mathfrak{s}_{t}^{*}$, there is only one principal orbit type, and the union of the principal orbits forms a dense open subset of $\mathfrak{s}_{t}^{*}$ whose complement has codimension $\geqq 2$. See [5, Chapter 4, Section 3] for a complete treatment of this material, or [10, Part II, Chapter 3, Section 1] modulo references to [5], or [18, Cap. 5] for a basic treatment, still with some references to [5].

The action of $M$ on $\mathfrak{s}^{*}$ commutes with dilation so the structural results on the $\mathfrak{s}_{t}$ also hold on $\mathfrak{s}^{*}=\bigcup_{t>0} \mathfrak{s}_{t}^{*}$. Define the Pf-nonsingular principal orbit set as follows: 


$$
\mathfrak{u}^{*}=\left\{\lambda \in \mathfrak{t}^{*} \mid \operatorname{Ad}^{*}(M) \lambda \text { is a principal } M \text {-orbit on } \mathfrak{s}^{*}\right\} .
$$

Now principal orbit set $\mathfrak{u}^{*}$ is a dense open set with complement of codimension $\geqq 2$ in $\mathfrak{s}^{*}$. If $\lambda \in \mathfrak{u}^{*}$ and $c \neq 0$ then $c \lambda \in \mathfrak{u}^{*}$ with isotropy $M_{c \lambda}=M_{\lambda}$. If $\lambda \in \mathfrak{u}_{t}^{*}:=$ $\mathfrak{u}^{*} \cap \mathfrak{s}_{t}^{*}$, so $\operatorname{Ad}^{*}(M) \lambda$ is a Pf-nonsingular principal orbit of $M$ on the sphere $\mathfrak{s}_{t}^{*}$, then $\operatorname{Ad}^{*}\left(M^{0}\right) \lambda$ is a principal orbit of $M^{0}$ on $\mathfrak{s}_{t}^{*}$. Principal orbit isotropy subgroups of compact connected linear groups are studied in [11] and the possibilities for the isotropy $\left(M^{0}\right)_{\lambda}$ are essentially known. The following lets us go from $\left(M^{0}\right)_{\lambda}$ to $M_{\lambda}$.

Proposition 7.1. ([29]) Suppose that $G$ is connected and linear. Then $M=F Z_{G} M^{0}$ where $Z_{G}$ is the center of $G, F=(\exp (i \mathfrak{a}) \cap K)$ is an elementary abelian 2-group, and $\mathrm{Ad}^{*}(F)$ acts trivially on $\mathfrak{s}^{*}$. If $\lambda \in \mathfrak{u}^{*}$ then the isotropy $M_{\lambda}=F Z_{G}\left(M^{0}\right)_{\lambda}$.

Thus the groups $M_{\lambda}$ are specified by the work of W.-C. and W.-Y. Hsiang [11].

Given $\lambda \in \mathfrak{u}^{*}$ the stepwise square integrable representation $\pi_{\lambda} \in \widehat{N}$ one proves that the Mackey obstruction $\varepsilon \in H^{2}\left(M_{\lambda} ; U(1)\right)$ is trivial, and in fact that $\pi_{\lambda}$ extends to a unitary representation $\pi_{\lambda}^{\dagger}$ of $N \rtimes M_{\lambda}$ on the representation space of $\pi_{\lambda}$.

Each $\lambda \in \mathfrak{u}^{*}$ now defines classes

$$
\mathscr{E}(\lambda):=\left\{\pi_{\lambda}^{\dagger} \otimes \gamma \mid \gamma \in \widehat{M_{\lambda}}\right\}, \mathscr{F}(\lambda):=\left\{\operatorname{Ind}_{N M_{\lambda}}^{N M}\left(\pi_{\lambda}^{\dagger} \otimes \gamma\right) \mid \pi_{\lambda}^{\dagger} \otimes \gamma \in \mathscr{E}(\lambda)\right\}
$$

of irreducible unitary representations of $N \rtimes M_{\lambda}$ and $N M$. The Mackey little group method, plus the fact that the Plancherel density on $\widehat{N}$ is polynomial on $\mathfrak{s}^{*}$, and $\mathfrak{s}^{*} \backslash \mathfrak{u}^{*}$ has measure 0 in $\mathfrak{t}^{*}$, gives us

Proposition 7.2. Plancherel measure for NM is concentrated on $\bigcup_{\lambda \in \mathfrak{u}^{*}} \mathscr{F}(\lambda)$, equivalence classes of irreducible representations $\eta_{\lambda, \gamma}:=\operatorname{Ind}_{N M_{\lambda}}^{N M}\left(\pi_{\lambda}^{\dagger} \otimes \gamma\right)$ such that $\pi_{\lambda}^{\dagger} \otimes \gamma \in \mathscr{E}(\lambda)$ and $\lambda \in \mathfrak{u}^{*}$. Further

$$
\left.\eta_{\lambda, \gamma}\right|_{N}=\left.\left(\operatorname{Ind}_{N M_{\lambda}}^{N M}\left(\pi_{\lambda}^{\dagger} \otimes \gamma\right)\right)\right|_{N}=\int_{M / M_{\lambda}}(\operatorname{dim} \gamma) \pi_{\mathrm{Ad}^{*}(m) \lambda} d\left(m M_{\lambda}\right)
$$

There is a Borel section $\sigma$ to $\mathfrak{u}^{*} \rightarrow \mathfrak{u}^{*} / \operatorname{Ad}^{*}(M)$ that picks out an element in each $M$-orbit so that $M$ has the same isotropy subgroup at each of those elements. In other words in each $M$-orbit on $\mathfrak{u}^{*}$ we measurably choose an element $\lambda=\sigma\left(\operatorname{Ad}^{*}(M) \lambda\right)$ such that those isotropy subgroups $M_{\lambda}$ are all the same. Let us denote

$$
M_{\diamond} \text { : isotropy subgroup of } M \text { at } \sigma\left(\operatorname{Ad}^{*}(M) \lambda\right) \text { for every } \lambda \in \mathfrak{u}^{*}
$$

We replace $M_{\lambda}$ by $M_{\diamond}$, independent of $\lambda \in \mathfrak{u}^{*}$, in Proposition 7.2. That lets us assemble to representations of Proposition 7.2 for a Plancherel Formula, as follows. Since $M$ is compact, we have the Schwartz space $\mathscr{C}(N M)$ just as in the discussion of $\mathscr{C}(N)$ between (6) and Theorem 3.5, except that the pullback exp* $\mathscr{C}(N M) \neq$ $\mathscr{C}(\mathfrak{n}+\mathfrak{m})$. The same applies to $\mathscr{C}(N A)$ and $\mathscr{C}(N A M)$ 
Proposition 7.3. Let $f \in \mathscr{C}(N M)$ and write $\left(f_{m}\right)(n)=f(n m)=\left({ }_{n} f\right)(m)$ for $n \in N$ and $m \in M$. The Plancherel density at $\operatorname{Ind}_{N M_{\diamond}}^{N M}\left(\pi_{\lambda}^{\dagger} \otimes \gamma\right)$ is $(\operatorname{dim} \gamma)\left|\operatorname{Pf}\left(b_{\lambda}\right)\right|$ and the Plancherel Formula for NM is

$$
f(n m)=c \int_{\mathfrak{u}^{*} / \operatorname{Ad}^{*}(M)} \sum_{\mathscr{F}(\lambda)} \operatorname{trace} \eta_{\lambda, \gamma}\left(n f_{m}\right) \cdot \operatorname{dim}(\gamma) \cdot\left|\operatorname{Pf}\left(b_{\lambda}\right)\right| d \lambda
$$

where $c=2^{d_{1}+\cdots+d_{m}} d_{1} ! d_{2} ! \ldots d_{m} !$, from $[6$, as in Theorem 3.5

\section{Minimal Parabolics: $M A N$ and $A N$.}

Let $G$ be a separable locally compact group of type I. Then [14, $\S 1]$ the Plancherel formula for $G$ has form

$$
f(x)=\int_{\widehat{G}} \operatorname{trace} \pi(D(r(x) f)) d \mu_{G}(\pi)
$$

where $D$ is an invertible positive self adjoint operator on $L^{2}(G)$, conjugation-semiinvariant of weight equal to the modular function $\delta_{G}$, and $\mu$ is a positive Borel measure on the unitary dual $\widehat{G}$. If $G$ is unimodular then $D$ is the identity and (23) reduces to the usual Plancherel formula. The point is that semi-invariance of $D$ compensates any lack of unimodularity. See [14, $\S 1]$ for a detailed discussion. $D \otimes \mu$ is unique (up to normalization of Haar measures) and one tries to find a "best" choice of $D$. Given any such pair $(D, \mu)$ we refer to $D$ as a Dixmier-Pukánszky Operator on $G$ and to $\mu$ as the associated Plancherel measure on $\widehat{G}$. We will construct a Dixmier-Pukánszky Operator from the Pfaffian polynomial $\operatorname{Pf}\left(b_{\lambda}\right)$.

Let $\delta_{A N}$ and $\delta_{Q}$ denote the modular functions on $A N$ and on $Q=M A N$. As $M$ is compact and $\operatorname{Ad}_{Q}(N)$ is unipotent on $\mathfrak{p}$, they are determined by their restrictions to $A$, where they are given by $\delta(\exp (\xi))=\exp (\operatorname{trace}(\operatorname{ad}(\xi)))$ with $\xi=\log a \in \mathfrak{a}$.

Lemma 8.1. Let $\xi \in \mathfrak{a}$. Then $\frac{1}{2}\left(\operatorname{dim} \mathfrak{l}_{r}+\operatorname{dim} \mathfrak{z}_{r}\right) \in \mathbb{Z}$ for $1 \leqq r \leqq m$ and

(i) the trace of $\operatorname{ad}(\xi)$ on $\mathfrak{l}_{r}$ is $\frac{1}{2}\left(\operatorname{dim} \mathfrak{l}_{r}+\operatorname{dim} \mathfrak{z}_{r}\right) \beta_{r}(\xi)$,

(ii) the trace of $\operatorname{ad}(\xi)$ on $\mathfrak{n}$ and on $\mathfrak{p}$ is $\frac{1}{2} \sum_{r}\left(\operatorname{diml}_{r}+\operatorname{dim}_{\mathfrak{z} r}\right) \beta_{r}(\xi)$,

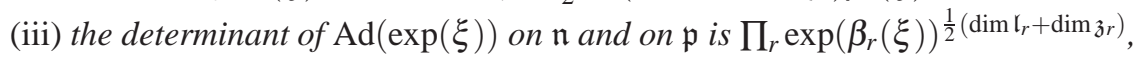
(iv) $\delta_{Q}($ man $)=\prod_{r} \exp \left(\beta_{r}(\log a)\right)^{\frac{1}{2}\left(\operatorname{dim} \mathfrak{l}_{r}+\operatorname{dim}_{\mathfrak{z} r}\right)}$ and $\delta_{A N}=\left.\delta_{Q}\right|_{A N}$.

Now compute

Lemma 8.2. Let $\xi \in \mathfrak{a}$ and $a=\exp (\xi) \in A$. Then $\operatorname{ad}(\xi) \operatorname{Pf}=\left(\frac{1}{2} \sum_{r} \operatorname{dim}\left(\mathfrak{l}_{r} / \mathfrak{z}_{r}\right) \beta_{r}(\xi)\right)$ Pf and $\operatorname{Ad}(a) \operatorname{Pf}=\left(\prod_{r} \exp \left(\beta_{r}(\xi)\right)^{\frac{1}{2} \operatorname{dim}\left(\mathfrak{l}_{r} / \operatorname{dim} \mathfrak{z}_{r}\right)}\right) \mathrm{Pf}$.

At this point it is convenient to introduce some notation and definitions.

Definition 8.3. The algebra $\mathfrak{s}$ is the quasi-center of $\mathfrak{n}$. The polynomial function

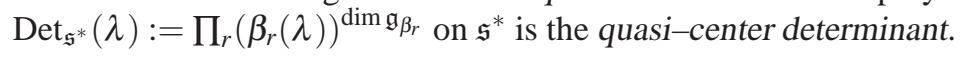


For $\xi \in \mathfrak{a}$ and $a=\exp (\xi) \in A$ compute $\left(\operatorname{Ad}(a) \operatorname{Det}_{\mathfrak{s}^{*}}\right)(\lambda)=\operatorname{Det}_{\mathfrak{s}^{*}}\left(\operatorname{Ad}^{*}\left(a^{-1}\right)(\lambda)\right)$ $=\prod_{r}\left(\beta_{r}\left(\operatorname{Ad}\left(a^{-1}\right)^{*} \lambda\right)\right)^{\operatorname{dim} \mathfrak{g}_{\beta_{r}}}=\prod_{r}\left(\beta_{r}\left(\exp \left(\beta_{r}(\xi)\right) \lambda\right)\right)^{\operatorname{dim} \mathfrak{g}_{\beta_{r}}}$. In other words,

Lemma 8.4. Let $a=\exp (\xi) \in A$. Then $\operatorname{Ad}(a) \operatorname{Det}_{\mathfrak{s}^{*}}=\left(\prod_{r} \exp \left(\beta_{r}(\xi)\right)^{\operatorname{dim}_{\mathfrak{z}} r}\right) \operatorname{Det}_{\mathfrak{s}^{*}}$ where $\xi=\log a \in \mathfrak{a}$.

Combining Lemmas $8.1,8.2$ and 8.4 we have

Proposition 8.5. The product $\mathrm{Pf} \cdot \operatorname{Det}_{\mathfrak{s}^{*}}$ is an $\operatorname{Ad}(M A N)$-semi-invariant (and thus $\operatorname{Ad}(A N)$-semi-invariant) polynomial on $\mathfrak{s}^{*}$ of degree $\frac{1}{2}(\operatorname{dim} \mathfrak{n}+\operatorname{dim} \mathfrak{s})$ and of weight equal to the respective modular functions of $Q$ and $A N$.

From $\mathfrak{n}=\mathfrak{v}+\mathfrak{s}$ we have $N=V S$ where $V=\exp (\mathfrak{v})$ and $S=\exp (\mathfrak{s})$. Now define

$D$ : Fourier transform of Pf $\cdot \operatorname{Det}_{\mathfrak{s}^{*}}$, acting on the $S$ variable of $N=V S$.

Theorem 8.6. The operator $D$ of (24) is an invertible self-adjoint differential operator of degree $\frac{1}{2}(\operatorname{dim} \mathfrak{n}+\operatorname{dim} \mathfrak{s})$ on $L^{2}(M A N)$ with dense domain $\mathscr{C}(M A N)$, and it is $\operatorname{Ad}(M A N)$-semi-invariant of weight equal to the modular function $\delta_{M A N}$. In other words $|D|$ is a Dixmier-Pukanszky Operator on MAN with domain equal to the space of rapidly decreasing $C^{\infty}$ functions. This applies as well to AN.

Since $\lambda \in \mathfrak{t}^{*}$ has nonzero projection on each summand $\mathfrak{z}_{r}^{*}$ of $\mathfrak{s}^{*}$, and $a \in A$ acts by the positive real scalar $\exp \left(\beta_{r}(\log (a))\right)$ on $\mathfrak{z} r$,

$$
A_{\lambda}=\exp \left(\left\{\xi \in \mathfrak{a} \mid \operatorname{each} \beta_{r}(\xi)=0\right\}\right), \text { independent of } \lambda \in \mathfrak{t}^{*} .
$$

Because of this independence, and using $\mathfrak{a}_{\diamond}=\left\{\xi \in \mathfrak{a} \mid\right.$ each $\left.\beta_{r}(\xi)=0\right\}$, we define

$$
A_{\diamond}=A_{\lambda} \text { for any (and thus for all) } \lambda \in \mathfrak{t}^{*} .
$$

Lemma 8.7. If $\lambda \in \sigma\left(\mathfrak{u}^{*}\right)$ then the stabilizer $(M A)_{\lambda}=M_{\diamond} A_{\diamond}$.

There is no problem with the Mackey obstruction:

Lemma 8.8. Let $\lambda \in \sigma\left(\mathfrak{u}^{*}\right)$. Recall the extension (before (21)) $\pi_{\lambda}^{\dagger}$ of $\pi_{\lambda}$ to $N M_{\diamond}$. Then $\pi_{\lambda}^{\dagger}$ extends to $\widetilde{\pi_{\lambda}} \in \widehat{N M_{\diamond} A_{\diamond}}$ with the same representation space as $\pi_{\lambda}$.

When $\lambda \in \sigma\left(\mathfrak{u}^{*}\right), \widehat{A_{\diamond}}$ consists of the unitary characters $\exp (i \phi): a \mapsto e^{i \phi(\log a)}$ with $\phi \in \mathfrak{a}_{\diamond}^{*}$. The representations of $Q$ corresponding to $\lambda$ are the

$$
\pi_{\lambda, \gamma, \phi}:=\operatorname{Ind}_{N M_{\diamond} A_{\diamond}}^{N M A}\left(\widetilde{\pi_{\lambda}} \otimes \gamma \otimes \exp (i \phi)\right) \text { where } \gamma \in \widehat{M_{\diamond}} \text { and } \phi \in \mathfrak{a}_{\diamond}^{*} .
$$

$\operatorname{Ad}^{*}(A)$ fixes $\gamma$ because $A$ centralizes $M$, and it fixes $\phi$ because $A$ is commutative, so

$$
\pi_{\lambda, \gamma, \phi} \cdot \operatorname{Ad}\left((m a)^{-1}\right)=\pi_{\mathrm{Ad}^{*}(m a) \lambda, \gamma, \phi}
$$


Proposition 8.9. Plancherel measure for $Q$ is concentrated on the the set of all $\pi_{\lambda, \gamma, \phi}$ for $\lambda \in \sigma\left(\mathfrak{u}^{*}\right), \gamma \in \widehat{M_{\diamond}}$ and $\phi \in \mathfrak{a}_{\diamond}^{*}$. The equivalence class of $\pi_{\lambda, \gamma, \phi}$ depends only on $\left(\operatorname{Ad}^{*}(M A) \lambda, \gamma, \phi\right)$.

Representations of $A N$ are the case $\gamma=1$. In effect, let $\pi_{\lambda}^{\prime}$ denote the obvious extension $\left.\widetilde{\pi_{\lambda}}\right|_{A N}$ of the stepwise square integrable representation $\pi_{\lambda}$ from $N$ to $N A_{\diamond}$ where $\widetilde{\pi_{\lambda}}$ is given by Lemma 8.8 . Denote

$$
\pi_{\lambda, \phi}=\operatorname{Ind}_{N A_{\diamond}}^{N A}\left(\pi_{\lambda}^{\prime} \otimes \exp (i \phi)\right) \text { where } \lambda \in \mathfrak{u}^{*} \text { and } \phi \in \mathfrak{a}_{\diamond}^{*} .
$$

Corollary 8.10. Plancherel measure for $A N$ is concentrated on the set of all $\pi_{\lambda, \phi}$ for $\lambda \in \mathfrak{u}^{*}$ and $\phi \in \mathfrak{a}_{\diamond}^{*}$. The equivalence class of $\pi_{\lambda, \phi}$ depends only on $\left(\operatorname{Ad}^{*}(M A) \lambda, \phi\right)$.

A result of C.C. Moore implies

Lemma 8.11. The $\mathrm{Pf}-$ nonsingular principal orbit set $\mathfrak{u}^{*}$ is a finite union of open $\operatorname{Ad}^{*}(M A)-$ orbits.

Let $\left\{\mathscr{O}_{1}, \ldots \mathscr{O}_{v}\right\}$ denote the (open) $\mathrm{Ad}^{*}(M A)$-orbits on $\mathfrak{u}^{*}$. Denote $\lambda_{i}=\sigma\left(\mathscr{O}_{i}\right)$, so $\mathscr{O}_{i}=\operatorname{Ad}^{*}(M A) \lambda_{i}$ and $(M A)_{\lambda_{i}}=M_{\diamond} A_{\diamond}$ for $1 \leqq i \leqq v$. Then Proposition 8.9 becomes

Theorem 8.12. Plancherel measure for MAN is concentrated on the set (of equivalence classes of ) unitary representations $\pi_{\lambda_{i}, \gamma, \phi}$ for $1 \leqq i \leqq \nu, \gamma \in \widehat{M_{\diamond}}$ and $\phi \in \mathfrak{a}_{\diamond}^{*}$.

Now the Plancherel Theorem for $Q=M A N$ is

The Plancherel Formula (or Fourier Inversion Formula) for MAN is

Theorem 8.13. Let $Q=M A N$ be a minimal parabolic subgroup of the real reductive Lie group G. Given $\pi_{\lambda, \gamma, \phi} \in \widehat{M A N}$ as described in (27) let $\Theta_{\pi_{\lambda, \gamma, \phi}}: h \mapsto$ trace $\pi_{\lambda, \gamma, \phi}(h)$ denote its distribution character. Then $\Theta_{\pi_{\lambda, \gamma, \phi}}$ is a tempered distribution. If $f \in \mathscr{C}(M A N)$ then

$$
f(x)=c \sum_{i=1}^{v} \sum_{\gamma \in \widehat{M_{\diamond}}} \int_{\mathfrak{a}_{\diamond}^{*}} \Theta_{\pi_{\lambda_{i}, \gamma, \phi}}(D(r(x) f))\left|\operatorname{Pf}\left(b_{\lambda_{i}}\right)\right| \operatorname{dim} \gamma d \phi
$$

where $c>0$ depends on normalizations of Haar measures.

The Plancherel Theorem for $N A$ follows similar lines. For the main computation in the proof of Theorem 8.13 we omit $M$ and $\gamma$. That gives

$$
\int_{\mathfrak{a}_{\diamond}^{*}} \operatorname{trace} \pi_{\lambda_{0}, \phi}(D h) d \phi=\int_{\mathrm{Ad}^{*}(A) \lambda_{0}} \operatorname{trace} \pi_{\lambda}(h)\left|\operatorname{Pf}\left(b_{\lambda}\right)\right| d \lambda
$$

In order to go from an $\operatorname{Ad}^{*}(A) \lambda_{0}$ to an integral over $\mathfrak{u}^{*}$ we use $M$ to parameterize the space of $\operatorname{Ad}^{*}(A)$-orbits on $\mathfrak{u}^{*}$. If $\lambda \in \mathfrak{u}^{*}$ one proves $\operatorname{Ad}^{*}(A) \lambda \cap \operatorname{Ad}^{*}(M) \lambda=\{\lambda\}$. That leads to 
Proposition 8.14. Plancherel measure for NA is concentrated on the equivalence classes of representations $\pi_{\lambda, \phi}=\operatorname{Ind}_{N A_{\diamond}}^{N A}\left(\pi_{\lambda}^{\prime} \otimes \exp (i \phi)\right)$ where $\lambda \in S_{i}:=\operatorname{Ad}^{*}(M) \lambda_{i}$, $1 \leqq i \leqq v$, $\pi_{\lambda}^{\prime}$ extends $\pi_{\lambda}$ from $N$ to $N A_{\diamond}$ and $\phi \in \mathfrak{a}_{\diamond}^{*}$. Representations $\pi_{\lambda, \phi}$ and $\pi_{\lambda^{\prime}, \phi^{\prime}}$ are equivalent if and only if $\lambda^{\prime} \in \operatorname{Ad}^{*}(A) \lambda$ and $\phi^{\prime}=\phi$. Further, $\left.\pi_{\lambda, \phi}\right|_{N}=$ $\int_{a \in A / A \diamond} \pi_{\mathrm{Ad}^{*}(a) \lambda} d a$.

Theorem 8.15. Let $Q=$ MAN be a minimal parabolic subgroup of the real reductive Lie group G. If $\pi_{\lambda, \phi} \in \widehat{A N}$ let $\Theta_{\pi_{\lambda, \phi}}: h \mapsto$ trace $\pi_{\lambda, \phi}(h)$ denote its distribution character. Then $\Theta_{\pi_{\lambda, \phi}}$ is a tempered distribution. If $f \in \mathscr{C}(A N)$ then

$$
f(x)=c \sum_{i=1}^{v} \int_{\lambda \in \operatorname{Ad} *(M) \lambda_{i}} \int_{\mathfrak{a}_{\diamond}^{*}} \operatorname{trace} \pi_{\lambda, \phi}(D(r(x) f))\left|\operatorname{Pf}\left(b_{\lambda}\right)\right| d \lambda d \phi .
$$

where $c>0$ depends on normalizations of Haar measures.

\section{Parabolic Subgroups in General: the Nilradical.}

In Sections 7 and 8 we studied minimal parabolic subgroups $Q=M A N$ in simple Lie groups, along with certain of their subgroups $M N$ and $A N$. This section and the next form a glance at more general parabolics. This material is taken from [32], which is a work in progress, and is limited to the part that I've written down. We start with the structure of the nilradical.

The condition (c) of (5) does not always hold for nilradicals of parabolic subgroups. In this section and the next we weaken (5) to

$$
N=L_{1} L_{2} \ldots L_{m-1} L_{m} \text { where }
$$

(a) each $L_{r}$ has unitary representations with coefficients in $L^{2}\left(L_{r} / Z_{r}\right)$,

(b) each $N_{r}:=L_{1} L_{2} \ldots L_{r}=N_{r-1} \rtimes L_{r}$ semidirect,

(c) if $r \geqq s$ then $\left[\mathfrak{l}_{r}, \mathfrak{z}_{s}\right]=0$.

The conditions of (31) are sufficient to construct stepwise square integrable representations, but are not always sufficient to compute the Pfaffian that is the Plancherel density. So we refer to (5) as the strong computability condition and make make use of the weak computability condition

$$
\text { Let } \mathfrak{l}_{r}=\mathfrak{l}_{r}^{\prime} \oplus \mathfrak{l}_{r}^{\prime \prime} \text { where } \mathfrak{l}_{r}^{\prime \prime \prime} \subset \mathfrak{z}_{r} \text { and } \mathfrak{v}_{r} \subset \mathfrak{l}_{r}^{\prime} ; \text { then }\left[\mathfrak{l}_{r}, \mathfrak{l}_{s}\right] \subset \mathfrak{l}_{s}^{\prime \prime}+\mathfrak{v}_{s} \text { for } r>s \text {. }
$$

where we retain $\mathfrak{l}_{r}=\mathfrak{z}_{r}+\mathfrak{v}_{r}$ and $\mathfrak{n}=\mathfrak{s}+\mathfrak{v}$.

Consider an arbitrary parabolic subgroup of $G$. It contains a minimal parabolic $Q=M A N$. Let $\Psi$ denote the set of simple roots for the positive system $\Delta^{+}(\mathfrak{g}, \mathfrak{a})$. Then the parabolic subgroups of $G$ that contain $Q$ are in one to one correspondence with the subsets $\Phi \subset \Psi$, say $Q_{\Phi} \leftrightarrow \Phi$, as follows. Denote $\Psi=\left\{\psi_{i}\right\}$ and set 


$$
\begin{aligned}
& \Phi^{r e d}=\left\{\alpha=\sum_{\psi_{i} \in \Psi} n_{i} \psi_{i} \in \Delta(\mathfrak{g}, \mathfrak{a}) \mid n_{i}=0 \text { whenever } \psi_{i} \notin \Phi\right\} \\
& \Phi^{n i l}=\left\{\alpha=\sum_{\psi_{i} \in \Psi} n_{i} \psi_{i} \in \Delta^{+}(\mathfrak{g}, \mathfrak{a}) \mid n_{i}>0 \text { for some } \psi_{i} \notin \Phi\right\} .
\end{aligned}
$$

On the Lie algebra level, $\mathfrak{q}_{\Phi}=\mathfrak{m}_{\Phi}+\mathfrak{a}_{\Phi}+\mathfrak{n}_{\Phi}$ where

$\mathfrak{a}_{\Phi}=\{\xi \in \mathfrak{a} \mid \psi(\xi)=0$ for all $\psi \in \Phi\}=\Phi^{\perp}$,

$\mathfrak{m}_{\Phi}+\mathfrak{a}_{\Phi}$ is the centralizer of $\mathfrak{a}_{\Phi}$ in $\mathfrak{g}$, so $\mathfrak{m}_{\Phi}$ has root system $\Phi^{\text {red }}$, and

$\mathfrak{n}_{\Phi}=\sum_{\alpha \in \Phi^{n i l}} \mathfrak{g}_{\alpha}$, nilradical of $\mathfrak{q}_{\Phi}$, sum of the positive $\mathfrak{a}_{\Phi^{-}}$-root spaces.

Since $\mathfrak{n}=\sum_{r} \mathfrak{l}_{r}$, as given in 17) and 18) we have

$$
\mathfrak{n}_{\Phi}=\sum_{r}\left(\mathfrak{n}_{\Phi} \cap \mathfrak{l}_{r}\right)=\sum_{r}\left(\left(\mathfrak{g}_{\beta_{r}} \cap \mathfrak{n}_{\Phi}\right)+\sum_{\Delta_{r}^{+}}\left(\mathfrak{g}_{\alpha} \cap \mathfrak{n}_{\Phi}\right)\right) .
$$

As ad( $\mathfrak{m})$ is irreducible on each restricted root space, if $\alpha \in\left\{\beta_{r}\right\} \cup \Delta_{r}^{+}$then $\mathfrak{g}_{\alpha} \cap \mathfrak{n}_{\Phi}$ is 0 or all of $\mathfrak{g}_{\alpha}$.

Lemma 9.1. Suppose $\mathfrak{g}_{\beta_{r}} \cap \mathfrak{n}_{\Phi}=0$. Then $\mathfrak{l}_{r} \cap \mathfrak{n}_{\Phi}=0$.

Lemma 9.2. Suppose $\mathfrak{g}_{\beta_{r}} \cap \mathfrak{n}_{\Phi} \neq 0$. Define $J_{r} \subset \Delta_{r}^{+}$by $\mathfrak{l}_{r} \cap \mathfrak{n}_{\Phi}=\mathfrak{g}_{\beta_{r}}+\sum_{J_{r}} \mathfrak{g}_{\alpha}$. Decompose $J_{r}=J_{r}^{\prime} \cup J_{r}^{\prime \prime}$ where $J_{r}^{\prime}=\left\{\alpha \in J_{r} \mid \sigma_{r} \alpha \in J_{r}\right\}$ and $J_{r}^{\prime \prime}=\left\{\alpha \in J_{r} \mid \sigma_{r} \alpha \notin J_{r}\right\}$. Then $\mathfrak{g}_{\beta_{r}}+\sum_{J_{r}^{\prime \prime}} \mathfrak{g}_{\alpha}$ belongs to a single $\mathfrak{a}_{\Phi}$-root space in $\mathfrak{n}_{\Phi}$, i.e. $\left.\alpha\right|_{\mathfrak{a}_{\Phi}}=\left.\beta_{r}\right|_{\mathfrak{a}_{\Phi}}$, for every $\alpha \in J_{r}^{\prime \prime}$.

Lemma 9.3. Suppose $\mathfrak{l}_{r} \cap \mathfrak{n}_{\Phi} \neq 0$. Then the algebra $\mathfrak{l}_{r} \cap \mathfrak{n}_{\Phi}$ has center $\mathfrak{g}_{\beta_{r}}+$ $\sum_{J_{r}^{\prime \prime}} \mathfrak{g}_{\alpha}$, and $\left.\mathfrak{l}_{r} \cap \mathfrak{n}_{\Phi}=\left(\mathfrak{g}_{\beta_{r}}+\sum_{J_{r}^{\prime \prime}} \mathfrak{g}_{\alpha}\right)+\left(\sum_{J_{r}^{\prime}} \mathfrak{g}_{\alpha}\right)\right)$. Further, $\mathfrak{l}_{r} \cap \mathfrak{n}_{\Phi}=\left(\sum_{J_{r}^{\prime \prime}} \mathfrak{g}_{\alpha}\right) \oplus$ $\left(\mathfrak{g}_{\beta_{r}}+\left(\sum_{J_{r}} \mathfrak{g}_{\alpha}\right)\right)$ direct sum of ideals.

It will be convenient to define sets of simple $\mathfrak{a}_{\Phi}$-roots

$$
\Psi_{1}=\Psi \text { and } \Psi_{s+1}=\left\{\psi \in \Psi \mid\left\langle\psi, \beta_{i}\right\rangle=0 \text { for } 1 \leqq i \leqq s\right\} .
$$

Note that $\Psi_{r}$ is the simple root system for $\left\{\alpha \in \Delta^{+}(\mathfrak{g}, \mathfrak{a}) \mid \alpha \perp \beta_{i}\right.$ for $\left.i<r\right\}$.

Lemma 9.4. If $r>s$ then $\left[\mathfrak{l}_{r} \cap \mathfrak{n}_{\Phi}, \mathfrak{g}_{\beta_{s}}+\sum_{J_{s}^{\prime \prime}} \mathfrak{g}_{\alpha}\right]=0$.

For our dealings with arbitrary parabolics it is not sufficient to consider linear functionals on $\sum_{r} \mathfrak{g}_{\beta_{r}}$. Instead we have to look at linear functionals on $\sum_{r}\left(\mathfrak{g}_{\beta_{r}}+\right.$ $\left.\sum_{J_{r}^{\prime \prime}} \mathfrak{g}_{\alpha}\right)$. of the form $\lambda=\sum \lambda_{r}$ where $\lambda_{r} \in \mathfrak{g}_{\beta_{r}}^{*}$ such that $b_{\lambda_{r}}$ is nondegenerate on $\sum_{r} \sum_{J_{r}} \mathfrak{g}_{\alpha}$. We know that $(5 \mathrm{c})$ ) holds for the nilradical of the minimal parabolic $\mathfrak{q}$ that contains $\mathfrak{q}_{\Phi}$. In view of Lemma 9.4 it follows that $b_{\lambda}\left(\mathfrak{l}_{r}, \mathfrak{l}_{s}\right)=\lambda\left(\left[\mathfrak{l}_{r}, \mathfrak{l}_{s}\right]=0\right.$ for $r>s$. For this particular type of $\lambda$, the bilinear form $b_{\lambda}$ has kernel $\sum_{r}\left(\mathfrak{g}_{\beta_{s}}+\sum_{J_{s}^{\prime \prime}} \mathfrak{g}_{\alpha}\right)$ and is nondegenerate on $\sum_{r} \sum_{J_{r}^{\prime}} \mathfrak{g}_{\alpha}$. Then $N_{\Phi}=\left(L_{1} \cap N_{\Phi}\right)\left(L_{2} \cap N_{\Phi}\right) \ldots\left(L_{m} \cap N_{\Phi}\right)$ satisfies the first two conditions of (5). That is enough to carry out the construction of stepwise square integrable representations $\pi_{\lambda}$ of $N_{\Phi}$, but one needs to do more to deal with Pfaffian polynomials as in (5) ( c)) and (32). 
Let $I_{1}=\left\{i\left|\beta_{i}\right|_{\mathfrak{a}_{\Phi}}=\left.\beta_{q_{1}}\right|_{\mathfrak{a}_{\Phi}}\right\}$ where $q_{1}$ is the first index of (5) with $\left.\beta_{q_{1}}\right|_{\mathfrak{a}_{\Phi}} \neq 0$. Next, $I_{2}=\left\{i\left|\beta_{i}\right|_{\mathfrak{a}_{\Phi}}=\left.\beta_{q_{2}}\right|_{\mathfrak{a}_{\Phi}}\right\}$ where $q_{2}$ is the first index of (5) such that $q_{2} \notin I_{1}$ and $\left.\beta_{q_{2}}\right|_{\mathfrak{a}_{\Phi}} \neq 0$. Continuing as long as possible, $I_{k}=\left\{i\left|\beta_{i}\right|_{\mathfrak{a}_{\Phi}}=\left.\beta_{q_{k}}\right|_{\mathfrak{a}_{\Phi}}\right\}$ where $q_{k}$ is the first index of (5) such that $q_{k} \notin\left(I_{1} \cup \cdots \cup I_{k-1}\right)$ and $\left.\beta_{q_{k}}\right|_{\mathfrak{a}_{\Phi}} \neq 0$. Then $I_{1} \cup \cdots \cup I_{\ell}$ consists of all the indices $i$ for which $\left.\beta_{i}\right|_{\mathfrak{a}_{\Phi}} \neq 0$. For $1 \leqq j \leqq \ell$ define

$$
\mathfrak{l}_{\Phi, j}=\sum_{i \in I_{j}}\left(\mathfrak{l}_{i} \cap \mathfrak{n}_{\Phi}\right)=\left(\sum_{i \in I_{j}} \mathfrak{l}_{i}\right) \cap \mathfrak{n}_{\Phi} \text { and } \mathfrak{l}_{\Phi, j}^{\dagger}=\sum_{k \geqq j} \mathfrak{l}_{\Phi, k} .
$$

Lemma 9.5. If $k \geqq j$ then $\left[\mathfrak{l}_{\Phi, k}, \mathfrak{l}_{\Phi, j}\right] \subset \mathfrak{l}_{\Phi, j}$. For each index $j, \mathfrak{l}_{\Phi, j}$ and $\mathfrak{l}_{\Phi, j}^{\dagger}$ are subalgebras of $\mathfrak{n}_{\Phi}$ and $\mathfrak{l}_{\Phi, j}$ is an ideal in $\mathfrak{l}_{\Phi, j}^{\dagger}$.

Lemma 9.6. If $k>j$ then $\left[\mathfrak{l}_{\Phi, k}, \mathfrak{l}_{\Phi, j}\right] \cap \sum_{i \in I_{j}} \mathfrak{g}_{\beta_{i}}=0$.

In the notation of Lemma9.2 if $r \in I_{j}$ then

$$
\mathfrak{l}_{r} \cap \mathfrak{n}_{\Phi}=\mathfrak{l}_{r}^{\prime}+\mathfrak{l}_{r}^{\prime \prime} \text { where } \mathfrak{l}_{r}^{\prime}=\mathfrak{g}_{\beta_{r}}+\sum_{J_{r}} \mathfrak{g}_{\alpha} \text { and } \mathfrak{l}_{r}^{\prime \prime}=\sum_{J_{r}^{\prime \prime}} \mathfrak{g}_{\alpha} .
$$

For $1 \leqq j \leqq \ell$ define

$$
\mathfrak{z} \Phi, j=\sum_{i \in I_{j}}\left(\mathfrak{g}_{\beta_{i}}+\mathfrak{l}_{i}^{\prime \prime}\right)
$$

and decompose

$$
\mathfrak{l}_{\Phi, j}=\mathfrak{l}_{\Phi, j}^{\prime}+\mathfrak{l}_{\Phi, j}^{\prime \prime} \text { where } \mathfrak{l}_{\Phi, j}^{\prime}=\sum_{i \in I_{j}} \mathfrak{l}_{i}^{\prime} \text { and } \mathfrak{l}_{\Phi, j}^{\prime \prime}=\sum_{i \in I_{j}} \mathfrak{l}_{i}^{\prime \prime} .
$$

Lemma 9.7. Recall $\mathfrak{\Upsilon}_{\Phi, j}^{\dagger}=\sum_{k \geqq j} \mathfrak{l}_{\Phi, k}$ from (37). For each $j$, both $\mathfrak{z}_{\Phi, j}$ and $\mathfrak{l}_{\Phi, j}^{\prime \prime}$ are central ideals in $\mathfrak{l}_{\Phi, j}^{\dagger}$, and $\mathfrak{z} \Phi, j$ is the center of $\mathfrak{l}_{\Phi, j}$.

Decompose

$$
\mathfrak{n}_{\Phi}=\mathfrak{z}_{\Phi}+\mathfrak{v}_{\Phi} \text { where } \mathfrak{z}_{\Phi}=\sum_{j} \mathfrak{z}_{\Phi, j}, \mathfrak{v}_{\Phi}=\sum_{j} \mathfrak{v}_{\Phi, j} \text { and } \mathfrak{v}_{\Phi, j}=\sum_{i \in I_{j}} \sum_{\alpha \in J_{i}^{\prime}} \mathfrak{g}_{\alpha} .
$$

Then Lemma 9.7 gives us (32) for the $\mathfrak{l}_{\Phi, j}: \mathfrak{l}_{\Phi, j}=\mathfrak{l}_{\Phi, j}^{\prime} \oplus \mathfrak{l}_{\Phi, j}^{\prime \prime}$ with $\mathfrak{l}_{\Phi, j}^{\prime \prime} \subset \mathfrak{z} \Phi, j$ and $\mathfrak{v}_{\Phi, j} \subset \mathfrak{l}_{\Phi, j}^{\prime}$.

Lemma 9.8. For generic $\lambda_{j} \in \mathfrak{z}_{\Phi, j}^{*}$ the kernel of $b_{\lambda_{j}}$ on $\mathfrak{l}_{\Phi, j}$ is just $\mathfrak{z}_{\Phi, j}$, in other words $b_{\lambda_{j}}$ is is nondegenerate on $\mathfrak{v}_{\Phi, j} \simeq \mathfrak{l}_{\Phi, j} / \mathfrak{z} \Phi, j$. In particular $L_{\Phi, j}$ has square integrable representations.

Theorem 9.9. Let $G$ be a real reductive Lie group and $Q$ a real parabolic subgroup. Express $Q=Q_{\Phi}$ in the notation of (33) and 34). Then its nilradical $N_{\Phi}$ has decomposition $N_{\Phi}=L_{\Phi, 1} L_{\Phi, 2} \ldots L_{\Phi, \ell}$ that satisfies the conditions of (5) and (32) as follows. The center $Z_{\Phi, j}$ of $L_{\Phi, j}$ is the analytic subgroup for $\mathfrak{z} \Phi, j$ and 
(a) each $L_{\Phi, j}$ has unitary representations with coefficients in $L^{2}\left(L_{\Phi, j} / Z_{\Phi, j}\right)$,

(b) each $N_{\Phi, j}:=L_{\Phi, 1} L_{\Phi, 2} \ldots L_{\Phi, j}$ is a normal subgroup of $N_{\Phi}$ with $N_{\Phi, j}=N_{\Phi, j-1} \rtimes L_{\Phi, j}$ semidirect,

(c) $\left[\mathfrak{l}_{\Phi, k}, \mathfrak{z} \Phi, j\right]=0$ and $\left[\mathfrak{l}_{\Phi, k}, \mathfrak{l}_{\Phi, j}\right] \subset \mathfrak{v}_{\Phi, j}+\mathfrak{l}_{\Phi, j}^{\prime \prime}$ for $k>j$.

In particular $N_{\Phi}$ has stepwise square integrable representations relative to the decomposition $N_{\Phi}=L_{\Phi, 1} L_{\Phi, 2} \ldots L_{\Phi, \ell}$.

\section{Amenable Subgroups of Semisimple Lie Groups.}

In this section we apply the results of Section 9 to certain important subgroups of the parabolic $Q_{\Phi}=M_{\Phi} A_{\Phi} N_{\Phi}$, specifically its amenable subgroups $A_{\Phi} N_{\Phi}, U_{\Phi} N_{\Phi}$ and $U_{\Phi} A_{\Phi} N_{\Phi}$ where $U_{\Phi}$ is a maximal compact subgroup of $M_{\Phi}$.

The theory of the group $U_{\Phi} N_{\Phi}$ goes exactly as in Section 7 When $N_{\Phi}=$ $L_{\Phi, 1} L_{\Phi, 2} \ldots L_{\Phi, \ell}$ is weakly invariant we can proceed more or less as in [29]. The argument, but not the final result, will make use of

Definition 10.1. The decomposition $N_{\Phi}=L_{\Phi, 1} L_{\Phi, 2} \ldots L_{\Phi, \ell}$ of Theorem 9.9 is invariant if each $\operatorname{ad}\left(\mathfrak{m}_{\Phi}\right) \mathfrak{z} \Phi, j=\mathfrak{z} \Phi, j$, equivalently if each $\operatorname{Ad}\left(M_{\Phi}\right) \mathfrak{z} \Phi, j=\mathfrak{z} \Phi, j$, in other words whenever $\mathfrak{z} \Phi, j=\mathfrak{g}_{\left[\Phi, \beta_{j_{0}}\right]}$. The decomposition $N_{\Phi}=L_{\Phi, 1} L_{\Phi, 2} \ldots L_{\Phi, \ell}$ is weakly invariant if each $\operatorname{Ad}\left(U_{\Phi}\right) \mathfrak{z} \Phi, j=\mathfrak{z} \Phi, j$.

Set

$$
\mathfrak{r}_{\Phi}^{*}=\left\{\lambda \in \mathfrak{s}_{\Phi}^{*} \mid P(\lambda) \neq 0 \text { and } \operatorname{Ad}\left(U_{\Phi}\right) \lambda \text { is a principal } U_{\Phi} \text {-orbit on } \mathfrak{s}_{\Phi}^{*}\right\}
$$

Then $\mathfrak{r}_{\Phi}^{*}$ is dense, open and $U_{\Phi}$-invariant in $\mathfrak{s}_{\Phi}^{*}$. By definition of principal orbit the isotropy subgroups of $U_{\Phi}$ at the various points of $\mathfrak{r}_{\Phi}^{*}$ are conjugate, and we take a measurable section $\sigma$ to $\mathfrak{r}_{\Phi}^{*} \rightarrow U_{\Phi} \backslash \mathfrak{r}_{\Phi}^{*}$ on whose image all the isotropy subgroups are the same,

$$
U_{\Phi}^{\prime} \text { : isotropy subgroup of } U_{\Phi} \text { at } \sigma\left(U_{\Phi}(\lambda)\right) \text {, independent of } \lambda \in \mathfrak{r}_{\Phi}^{*}
$$

The principal isotropy subgroups $U_{\Phi}^{\prime}$ are pinned down in [11]. Given $\lambda \in \mathfrak{r}_{\Phi}^{*}$ and $\gamma \in \widehat{U_{\Phi}^{\prime}}$ let $\pi_{\lambda}^{\dagger}$ denote the extension of $\pi_{\lambda}$ to a representation of $U_{\Phi}^{\prime} N_{\Phi}$ on the space of $\pi_{\lambda}$ and define

$$
\pi_{\lambda, \gamma}=\operatorname{Ind}_{U_{\Phi}^{\prime} N_{\Phi}}^{U_{\Phi} N_{\Phi}}\left(\gamma \otimes \pi_{\lambda}^{\dagger}\right)
$$

The first result in this setting, as in [29, Proposition 3.3], is

Theorem 10.2. Suppose that $N_{\Phi}=L_{\Phi, 1} L_{\Phi, 2} \ldots L_{\Phi, \ell}$ as in 31. Then the Plancherel density on $\widehat{U_{\Phi} N_{\Phi}}$ is concentrated on the representations $\pi_{\lambda, \gamma}$ of [45], the Plancherel density at $\pi_{\lambda, \gamma}$ is $(\operatorname{dim} \gamma)|P(\lambda)|$, and the Plancherel Formula for $U_{\Phi} N_{\Phi}$ is 


$$
f(u n)=c \int_{\mathfrak{r}_{\Phi}^{*} / \mathrm{Ad}^{*}\left(U_{\Phi}\right)} \sum_{\gamma \in \widehat{U_{\Phi}^{\prime}}} \operatorname{trace} \operatorname{Ind}_{U_{\Phi}^{\prime} N_{\Phi}}^{U_{\Phi} N_{\Phi}} r_{u n}(f) \cdot \operatorname{dim}(\gamma) \cdot|P(\lambda)| d \lambda
$$

where $c=2^{d_{1}+\cdots+d_{\ell}} d_{1} ! d_{2} ! \ldots d_{\ell} !$ as in $(6)$.

Recall the notion of amenability.. A mean on a locally compact group $H$ is a linear functional $\mu$ on $L^{\infty}(H)$ of norm 1 and such that $\mu(f) \geqq 0$ for all real-valued $f \geqq 0$. $H$ is amenable if it has a left-invariant mean. Solvable groups and compact groups are amenable, as are extensions of amenable groups by amenable subgroups. In particular $E_{\Phi}:=U_{\Phi} A_{\Phi} N_{\Phi}$ and its closed subgroups are amenable.

We need a technical condition [15], p. 132]. Let $H$ be the group of real points in a linear algebraic group whose rational points are Zariski dense, let $A$ be a maximal $\mathbb{R}$-split torus in $H$, let $Z_{H}(A)$ denote the centralizer of $A$ in $H$, and let $H_{0}$ be the algebraic connected component of the identity in $H$. Then $H$ is isotropically connected if $H=H_{0} \cdot Z_{H}(A)$. More generally we will say that a subgroup $H \subset G$ is isotropically connected if the algebraic hull of $\operatorname{Ad}_{G}(H)$ is isotropically connected.

Proposition 10.2. [15, Theorem 3.2]. The groups $E_{\Phi}:=U_{\Phi} A_{\Phi} N_{\Phi}$ are maximal amenable subgroups of $G$. They are isotropically connected and self-normalizing. The various $\Phi \subset \Psi$ are mutually non-conjugate. An amenable subgroup $H \subset G$ is contained in some $E_{\Phi}$ if and only if it is isotropically connected.

The isotropy subgroups are the same at every $\lambda \in \mathfrak{t}_{\Phi}^{*}$,

$$
A_{\Phi}^{\prime} \text { : isotropy subgroup of } A_{\Phi} \text { at } \lambda \in \mathfrak{r}_{\Phi}^{*} .
$$

Given a stepwise square integrable representation $\pi_{\lambda}$ where $\lambda \in \mathfrak{s}_{\Phi}^{*}$, write $\pi_{\lambda}^{\dagger}$ for the extension of $\pi_{\lambda}$ to a representation of $A_{\Phi}^{\prime} N_{\Phi}$ on the same Hilbert space. That extension exists because the Mackey obstruction vanishes. The representations of $A_{\Phi}^{\prime} N_{\Phi}$ corresponding to $\pi_{\lambda}$ are the

$$
\pi_{\lambda, \phi}:=\operatorname{Ind}_{A_{\Phi}^{\prime} N_{\Phi}}^{A_{\Phi} N_{\Phi}}\left(\exp (i \phi) \otimes \pi_{\lambda}^{\dagger}\right) \text { where } \phi \in \mathfrak{a}_{\Phi}^{\prime} .
$$

Note also that

$$
\pi_{\lambda, \phi} \cdot \operatorname{Ad}(a n)=\pi_{\mathrm{Ad}^{*}(a) \lambda, \phi} \text { for } a \in A_{\Phi} \text { and } n \in N_{\Phi} .
$$

The resulting formula $f(x)=\int_{\widehat{H}} \operatorname{trace} \pi(D(r(x) f)) d \mu_{H}(\pi), H=A_{\Phi} N_{\Phi}$, is

Theorem 10.3. Let $Q_{\Phi}=M_{\Phi} A_{\Phi} N_{\Phi}$ be a parabolic subgroup of the real reductive Lie group G. Given $\pi_{\lambda, \phi} \in \widehat{A_{\Phi} N_{\Phi}}$ as described in 477, its distribution character $\Theta_{\pi_{\lambda, \phi}}: h \mapsto$ trace $\pi_{\lambda, \phi}(h)$ is a tempered distribution. If $f \in \mathscr{C}\left(A_{\Phi} N_{\Phi}\right)$ then

$$
f(x)=c \int_{\left(\mathfrak{a}_{\Phi}^{\prime}\right)^{*}}\left(\int_{\mathfrak{s}_{\Phi}^{*} / \mathrm{Ad}^{*}\left(A_{\Phi}\right)} \Theta_{\pi_{\lambda, \phi}}(D(r(x) f))\left|\operatorname{Pf}\left(b_{\lambda}\right)\right| d \lambda\right) d \phi
$$

where $c=2^{d_{1}+\cdots+d_{\ell}} d_{1} ! d_{2} ! \ldots d_{\ell} !$. 
The representations of $U_{\Phi} A_{\Phi} N_{\Phi}$ corresponding to $\pi_{\lambda}$ are the

$$
\pi_{\lambda, \phi, \gamma}:=\operatorname{Ind}_{U_{\Phi}^{\prime} A_{\Phi}^{\prime} N_{\Phi}}^{U_{\Phi} A_{\Phi} N_{\Phi}}\left(\gamma \otimes \exp (i \phi) \otimes \pi_{\lambda}^{\dagger}\right) \text { where } \phi \in \mathfrak{a}_{\Phi}^{\prime} \text { and } \gamma \in \widehat{U_{\Phi}^{\prime}} \text {. }
$$

Combining Theorems 10.2 and 10.3 we arrive at

Theorem 10.4. Let $Q_{\Phi}=M_{\Phi} A_{\Phi} N_{\Phi}$ be a parabolic subgroup of the real reductive Lie group $G$ and decompose $N_{\Phi}=L_{\Phi, 1} L_{\Phi, 2} \ldots L_{\Phi, \ell}$ as in (31). Then the Plancherel density on $\widehat{U}_{\Phi A_{\Phi} N}$ is concentrated on the $\pi_{\lambda, \phi, \gamma}$ of 4 49, the Plancherel density at $\pi_{\lambda, \phi, \gamma}$ is $(\operatorname{dim} \gamma)|P(\lambda)|$, the distribution character $\Theta_{\pi_{\lambda, \phi, \gamma}}: h \mapsto \operatorname{trace} \pi_{\lambda, \phi, \gamma}(h)$ is tempered, and if $f \in \mathscr{C}\left(U_{\Phi} A_{\Phi} N_{\Phi}\right)$ then

$$
f(x)=c \sum_{\widehat{U_{\Phi}^{\prime}}} \int_{\left(\mathfrak{a}_{\Phi}^{\prime}\right)^{*}}\left(\int_{\mathfrak{s}_{\Phi}^{*} / \operatorname{Ad}^{*}\left(U_{\Phi} A_{\Phi}\right)} \Theta_{\pi_{\lambda, \phi, \gamma}}(D(r(x) f)) \operatorname{deg}(\gamma)\left|\operatorname{Pf}\left(b_{\lambda}\right)\right| d \lambda\right) d \phi
$$

where $c=2^{d_{1}+\cdots+d_{\ell}} d_{1} ! d_{2} ! \ldots d_{\ell} !$.

\section{References}

1. L. Auslander et al, "Flows on Homogeneous Spaces", Ann. Math. Studies 53, 1963.

2. I. Beltita \& D. Beltita, Coadjoint orbits of stepwise square integrable representations, to appear. $\{$ arXiv: 1408.1857$\}$

3. I. Beltita \& J. Ludwig, Spectral synthesis for coadjoint orbits of nilpotent Lie groups, to appear. $\{$ arXiv:1412.6323\}

4. I. Beltita \& D. Beltita, Representations of nilpotent Lie groups via measurable dynamical systems $\{$ arXiv: 1510.05272$\}$

5. G. Bredon, Introduction to Compact Transformation Groups, Academic Press, 1972.

6. W. Casselman, Introduction to the Schwartz space of $\Gamma \backslash G$, Canadian J. Math. 40 (1989), 285-320.

7. M. Duflo, Sur les extensions des représentations irréductibles des groups de Lie nilpotents, Ann. Sci. de 1' École Norm. Supér., 4ième série 5 (1972), 71-120.

8. I. Dimitrov, I. Penkov \& J. A. Wolf, A Bott-Borel-Weil theory for direct limits of algebraic groups, Amer. J of Math. 124 (2002), 955-998.

9. J. Faraut, Infinite dimensional harmonic analysis and probability, in "Probability Measures on Groups: Recent Directions and Trends," ed. S. G. Dani \& P. Graczyk, Narosa, New Delhi, 2006.

10. V. V. Gorbatsevich, A. L. Onishchik \& E. B. Vinberg, Foundations of Lie Theory and Lie Transformation Groups, Springer, 1997.

11. W.-C. Hsiang \& W.-Y. Hsiang, Differentiable actions of compact connected classical groups II, Annals of Math. 92 (1970), 189-223.

12. A. A. Kirillov, Unitary representations of nilpotent Lie groups, Uspekhi Math. Nauk 17 (1962), 57-110 (English: Russian Math. Surveys 17 (1962), 53-104).

13. M. Krämer, Sphärische Untergruppen in kompakten zusammenhängenden Liegruppen, Compositio Math. 38 (1979), 129-153.

14. R. L. Lipsman \& J. A. Wolf, The Plancherel formula for parabolic subgroups of the classical groups, Journal D’Analyse Mathématique, 34 (1978), 120-161.

15. C. C. Moore, Amenable subgroups of semi-simple Lie groups and proximal flows. Israel J. Math. 34 (1979), 121-138. 
16. C. C. Moore, Decomposition of unitary representations defined by discrete subgroups of nilpotent groups, Ann. Math. 82 (1965), 146-182

17. C. C. Moore \& J. A. Wolf, Square integrable representations of nilpotent groups. Transactions of the American Mathematical Society, 185 (1973), 445-462.

18. S. de Neymet Urbina (con la colaboración de R. Jiménez Benítez), Introducción a los Grupos Topológicos de Transformaciones, Sociedad Matemática Mexicana, 2005.

19. G. I. Ol'shanskii, Unitary representations of infinite dimensional pairs $(G, K)$ and the formalism of R. Howe, in "Representations of Lie Groups and Related Topics, ed. A. M. Vershik \& D. P. Zhelobenko," Advanced Studies Contemp. Math. 7, Gordon \& Breach, 1990.

20. L. Pukánszky, On characters and the Plancherel formula of nilpotent groups, J. Functional Analysis 1 (1967), 255-280.

21. M. S. Raghunathan, "Discrete Subgroups of Lie Groups", Ergebnisse der Mathematik und ihrer Grenzgebeite 68, 1972.

22. E. B. Vinberg, Commutative homogeneous spaces and co-isotropic symplectic actions, Russian Math. Surveys 56 (2001), 1-60.

23. E. B. Vinberg, Commutative homogeneous spaces of Heisenberg type, Trans Moscow Math. Soc. 64 (2003), 45-78.

24. J. A. Wolf, Classification and Fourier inversion for parabolic subgroups with square integrable nilradical. Memoirs of the American Mathematical Society, Number 225, 1979.

25. J. A. Wolf, Direct limits of principal series representations, Compositio Mathematica, 141 (2005), 1504-1530.

26. J. A. Wolf, Harmonic Analysis on Commutative Spaces, Math. Surveys \& Monographs vol. 142, Amer. Math. Soc., 2007.

27. J. A. Wolf, Infinite dimensional multiplicity free spaces II: Limits of commutative nilmanifolds, to appear.

28. J. A. Wolf, Stepwise square integrable representations of nilpotent Lie groups, Mathematische Annalen vol. 357 (2013), pp. 895-914. \{arXiv: see 1212.1908\}

29. J. A. Wolf, The Plancherel Formula for Minimal Parabolic Subgroups, Journal of Lie Theory, vol. 24 (2014), pp. 791-808. \{arXiv: 1306.6392 (math RT)\}

30. J. A. Wolf, Stepwise square integrable representations for locally nilpotent Lie groups, Transformation Groups, vol. 20 (2015), pp. 863-879. \{arXiv:1402.3828 (math RT, math FA)\}

31. J. A. Wolf, On the analytic structure of commutative nilmanifolds, The Journal of Geometric Analysis, to appear. \{arXiv:1407.0399 (math RT, math DG)\}

32. J. A. Wolf, Stepwise square integrability for nilradicals of parabolic subgroups and maximal amenable subgroups, work in progress.

33. O. S. Yakimova, Weakly symmetric riemannian manifolds with reductive isometry group, Math. USSR Sbornik 195 (2004), 599-614.

34. O. S. Yakimova, "Gelfand Pairs," Bonner Math. Schriften (Universität Bonn) 374, 2005.

35. O. S. Yakimova, Principal Gelfand pairs, Transformation Groups 11 (2006), 305335. 